\title{
Physics implications of flat directions in free fermionic superstring models. II. Renormalization group analysis
}

\author{
G. Cleaver, ${ }^{1, *}$ M. Cvetič,${ }^{1}$ J. R. Espinosa, ${ }^{2}$ L. Everett, ${ }^{1,3}$ P. Langacker, ${ }^{1}$ and J. Wang ${ }^{1}$ \\ ${ }^{1}$ Department of Physics and Astronomy, University of Pennsylvania, Philadelphia, Pennsylvania 19104-6396 \\ ${ }^{2}$ CERN, TH Division, CH-1211 Geneva 23, Switzerland \\ ${ }^{3}$ Randall Laboratory of Physics, University of Michigan, Ann Arbor, Michigan 48109
}

(Received 30 November 1998; published 15 April 1999)

\begin{abstract}
We continue the investigation of the physics implications of a class of flat directions for a prototype quasi-realistic free fermionic string model (CHL5), building upon the results of a previous paper in which the complete mass spectrum and effective trilinear couplings of the observable sector were calculated to all orders in the superpotential. We introduce soft supersymmetry breaking mass parameters into the model, and investigate the gauge symmetry breaking patterns and the renormalization group analysis for two representative flat directions, which leave an additional $U(1)^{\prime}$ as well as the SM gauge group unbroken at the string scale. We study symmetry breaking patterns that lead to a phenomenologically acceptable $Z-Z^{\prime}$ hierarchy, $M_{Z^{\prime}}$ $\sim \mathcal{O}(1 \mathrm{TeV})$ and $10^{12} \mathrm{GeV}$ for electroweak and intermediate scale $U(1)^{\prime}$ symmetry breaking, respectively, and the associated mass spectra after electroweak symmetry breaking. The fermion mass spectrum exhibits unrealistic features, including massless exotic fermions, but has an interesting $d$-quark hierarchy and associated CKM matrix in one case. There are (some) non-canonical effective $\mu$ terms, which lead to a non-minimal Higgs sector with more than two Higgs doublets involved in the symmetry breaking, and a rich structure of Higgs particles, charginos, and neutralinos, some of which, however, are massless or ultralight. In the electroweak scale cases the scale of supersymmetry breaking is set by the $Z^{\prime}$ mass, with the sparticle masses in the several TeV range. [S0556-2821(99)06109-3]

PACS number(s): 12.60.Jv, 12.60.Cn, 14.80.Cp
\end{abstract}

\section{INTRODUCTION}

In recent work [1-3], techniques have been developed which set the stage for the "top-down" analysis of a class of quasi-realistic string models. Models in this class have $N$ $=1$ supersymmetry, the standard model (SM) gauge group as a part of the gauge structure, and candidate fields for the three generations of quarks and leptons as well as two electroweak Higgs doublets. Such quasi-realistic models have been constructed in weakly coupled heterotic superstring theory in a variety of constructions [4-9]; in particular, we consider a class of free fermionic models [6-9].

In general, these quasi-realistic free fermionic models have an extended gauge group [including a non-Abelian "hidden" sector gauge group and a number of additional $U(1)$ 's], and a large number of fields in addition to the minimal supersymmetric standard model (MSSM) fields, which include a number of non-Abelian singlets, fields which transform under the hidden sector gauge group, and SM exotics. These models also have the important property that the superpotential is calculable, in principle to all orders in the nonrenormalizable terms. The trilinear terms in the superpotential have large Yukawa couplings of $\mathcal{O}(g)$, where $g$ is the gauge coupling at the string scale. In contrast with general field-theoretic models, additional string (world sheet) sym-

\footnotetext{
*Present address: Center for Theoretical Physics, Texas A \& M University, College Station, Texas 77843-4242.
}

metries can forbid terms allowed by gauge invariance. ${ }^{1}$

These models generically possess an "anomalous" $U(1)$ at the level of the effective theory. The presence of the anomalous $U(1)$ leads to the generation of a FayetIliopoulos (FI) term at genus 1; this term triggers scalar fields to acquire string-scale vacuum expectation values (VEV's) along $D$ and $F$ flat directions, leading to a "restabilization" of the string vacuum. Thus, as the necessary first step in the analysis, we developed techniques [1] to classify the $D$ flat directions which can be proved to be $F$ flat to all orders of a general perturbative heterotic superstring model with an anomalous $U(1)$. For the sake of simplicity, we chose to consider flat directions formed of non-Abelian singlets only, and selected the singlet fields with zero hypercharge to preserve the SM gauge group. We applied our method to a prototype string model, model 5 of Chaudhuri, Hockney and Lykken (CHL5) [9] in [1], and more recently to a number of free fermionic string models in [2].

The next step in the analysis of this class of models is to analyze the effective theory along such flat directions. In general, the rank of the gauge group is reduced, and effective couplings are induced by the coupling of the fields in the flat direction to the rest of the fields in the model. The effective mass terms generated in this way will give some of the fields superheavy masses, so that they decouple from the theory at the string scale. In addition to the trilinear couplings of the

\footnotetext{
${ }^{1}$ For a review of the phenomenology of string models, see [10] and references therein.
} 
original superpotential, effective trilinear terms are generated from higher-dimensional terms for the remaining light fields; such couplings can have important implications for the phenomenology of the model. ${ }^{2}$

In a recent work [3] we studied in detail the physics implications of the effective theory after vacuum restabilization for a prototype model: model 5 of [9] (CHL5). The mass spectrum and the effective trilinear terms (in the observable sector) were calculated exactly at the string tree level and to all orders in the vacuum expectation value of the fields in the flat directions for all of the flat directions classified in [1]. However, we presented the detailed analysis for two representative flat directions, which encompass the general features of this class of flat directions. These two flat directions are part of a subset of flat directions of the model which break the maximal number of $U(1)$ 's, leaving an additional non-anomalous $U(1)^{\prime}$ as well as the SM gauge group unbroken at the string scale (as well as the hidden sector gauge groups). Importantly, the string world sheet symmetries forbid many of the gauge-allowed terms in the effective theory, which has a number of implications for the phenomenology of the model.

The calculation of the mass spectrum revealed that along with the MSSM fields, there remain a large number of massless exotic superfields at the string scale, due to the absence of the corresponding effective mass terms in the superpotential. These fields include additional electroweak doublets and electrically charged singlets, leading to a larger number of fields in the observable sector in this model than in the MSSM.

While the scalar fields can acquire masses from the supersymmetry breaking, their fermionic superpartners can remain light compared with the electroweak scale. We also found that many of the massless exotic fields do not couple directly to the observable sector fields at the order of the effective trilinear superpotential, such that they do not participate in the radiative electroweak and $U(1)^{\prime}$ gauge symmetry breaking. Therefore, there is no mechanism for these exotic fields to acquire masses. The additional massless particle content has an impact on gauge coupling unification, and renders the hidden sector gauge groups non-asymptotically free (such that there is no possibility of dynamical supersymmetry breaking in this sector due to strong coupling dynamics).

The presence of massless exotics seems to be generic and thus it is a serious obstacle for deriving phenomenologically acceptable physics from these models. Nevertheless, they have enough realistic features that it is worthwhile to study in detail the implications of the effective trilinear terms of

\footnotetext{
${ }^{2}$ The analysis of effective nonrenormalizable terms is deferred to further study. In this case complications arise due to the fact that the fourth order (nonrenormalizable) terms present in the original superpotential could be competitive in strength [11] with the trilinear ones, as well as generated in a number of other ways, such as via the decoupling of heavy states [12], a nonminimal Kähler potential, and the corrections to the Kähler potential due to the large VEV's. In the following we will not include such effects and assume a minimal Kähler potential.
}

the superpotential. These terms have a number of concrete implications for the observable sector physics. In particular, the effective third order superpotential does not have a canonical $\mu$ term (involving the standard electroweak Higgs doublets) in either example, but instead non-canonical $\mu$ terms involving some of the additional Higgs doublets. Such non-canonical terms suffice for the electroweak symmetry breaking. However, in these examples, there are not enough such terms to involve all of the Higgs doublets, resulting in massless charginos, neutralinos and an unwanted global $U(1)$. We found that when imposing the requirement that there be three lepton doublets in the model, $R$ parity is not conserved due to the presence of $L$-violating terms which leads to possible lightest supersymmetric particle (LSP) decay in both of the examples. The second representative flat direction also has $B$-violating terms (with implications for proton decay), as well as couplings which yield textures in the quark and lepton sectors.

The purpose of this paper is to take the phenomenological analysis of these models to the next stage: to determine the low energy implications of these string vacua for the gauge symmetry breaking patterns and the analysis of the low energy mass spectra. Specifically, we investigate in detail the nature of the electroweak and $U(1)^{\prime}$ gauge symmetry breaking patterns and the accompanying mass spectrum. Again, we choose to carry out this analysis for these two representative flat directions.

Such an analysis can only be performed after supersymmetry breaking has been incorporated. Since the origin of the supersymmetry breaking in string theory is not well understood, no quantitative, phenomenologically viable derivation of a supersmmetry breaking pattern is available. Thus we take a modest approach and parametrize the supersymmetry breaking by introducing soft supersymmetry breaking mass terms at the string scale. We can then proceed with the study of the renormalization group evolution of all the parameters in the observable sector and the study of their implications for the low-energy physics.

Although the mass spectrum and the effective trilinear superpotential for the representative flat directions of the CHL5 model are not realistic due to the additional massless exotics and the nature of the effective trilinear couplings (for details see [3]), we choose to focus on the light fields which participate in the gauge symmetry breaking, with the goal of obtaining scenarios with a realistic $Z-Z^{\prime}$ hierarchy. As shown in [13], in this class of models the breaking scale of the $U(1)^{\prime}$ can be either at the electroweak $(\mathrm{TeV})$ scale or at an intermediate scale, depending on the $U(1)^{\prime}$ charges and trilinear couplings of the massless SM singlet fields. In the first representative flat direction, the massless particle content at the string scale does not allow for the intermediate scale $U(1)^{\prime}$ breaking scenario, and hence the breaking is at the electroweak scale. However, both scenarios are possible for the second representative flat direction.

In this paper, we demonstrate these symmetry breaking scenarios explicitly for each of the two representative flat directions. Numerical results are presented for specific (typical) choices of the soft supersymmetry breaking terms at the string scale, which in turn yield a realistic $Z-Z^{\prime}$ hierarchy. 
We calculate the low energy spectrum explicitly with emphasis on the study of the Higgs boson mass spectrum and contrast its features with those of both the MSSM and the "bottom-up" analysis of the string models with an additional $U(1)^{\prime}$ studied in [14]. [For the first flat direction the complete mass spectrum, including that of the supersymmetric partners, is presented. For the second flat direction we specifically address the texture in the (bottom) quark sector.] Because of the fact that the number of Higgs fields participating in the symmetry breaking pattern is larger than that assumed in [14], the presence of some (but not all possible) non-canonical $\mu$ terms implies additional massless charginos, neutralinos, and Higgs bosons as well as new patterns in the massive Higgs spectrum.

The paper is structured as follows. In Sec. II, we summarize the features of the CHL5 model and define the two specific flat directions. In Sec. III, we present the mass spectrum and the effective trilinear superpotential couplings for the first flat direction of the CHL5 model. We demonstrate the possibility of realistic electroweak symmetry breaking scenarios of the SM gauge group along with the additional $U(1)^{\prime}$. In Sec. IV we demonstrate the $U(1)^{\prime}$ symmetry breaking scenarios at both the electroweak and intermediate scales for the second representative flat direction. Finally, in Sec. V we present the summary and conclusions.

\section{PRELIMINARIES}

The starting point of the analysis is the effective theory along the two representative flat directions of the CHL5 model [9]. In previous work, we have presented techniques for classifying the flat directions [1] and demonstrated the calculation of the effective couplings along these "restabilized vacua" of the model [3]. For the sake of completeness, we summarize the method and the results here, and refer the reader to $[1,3]$ for the details.

\section{A. Method}

In the class of quasi-realistic string models considered, there is an anomalous $U(1)$ generically present as part of the gauge structure, for which the anomalies are cancelled by the four-dimensional version of the Green-Schwarz mechanism. This standard anomaly cancellation mechanism leads to the generation of a nonzero FI contribution $\xi$ to the $D$ term of $U(1)_{A}$, with

$$
\xi=\frac{g_{\text {string }}^{2} M_{P}^{2}}{192 \pi^{2}} \operatorname{Tr} Q_{A},
$$

in which $g_{\text {string }}$ is related to the gauge coupling $g$ by the relation $g_{\text {string }}=g / \sqrt{2}[19]$ ( $g$ is normalized according to the standard [grand unified theory (GUT)] conventions, i.e., $\operatorname{Tr} T_{a} T_{b}=\delta_{a b} / 2$ for the generators of the fundamental representation of $S U(N))$ and $M_{P}=M_{P l} / \sqrt{8 \pi}$ is the reduced Planck mass, with $M_{P l} \sim 1.2 \times 10^{19} \mathrm{GeV}$. This term would appear to break supersymmetry in the original string vacuum. However, it triggers certain scalar fields to acquire
VEV's of $\mathcal{O}\left(M_{\text {String }}\right)$ along $D$ and $F$ flat directions, leading to a supersymmetric "restabilized" string vacuum.

Therefore, the classification of the flat directions is the necessary first step in the analysis of the string model. In [1], we presented techniques to classify a subset of $D$ flat directions which can be proved to be $F$ flat to all orders in the superpotential. For the sake of simplicity and to preserve the SM gauge group at the string scale, we chose to analyze flat directions formed from non-Abelian singlet fields with zero hypercharge. In general, the FI term sets the scale of the VEV's in the flat direction, although in some cases some of the VEV's are undetermined (but bounded from above).

The next stage of the analysis is to determine the effective theory along such flat directions [3]. In general, the rank of the gauge group will be reduced [i.e., several $U(1)$ 's will be broken]. Effective couplings can be generated from higherdimensional operators in the superpotential after replacing the fields in the flat direction by their VEV's. In particular, for a given flat direction $P$, effective mass terms for the fields $\Psi_{i}, \Psi_{j}\left(\Psi_{i, j} \notin\left\{\Phi_{k} \in P\right\}\right)$ may be generated via

$$
W \sim \Psi_{i} \Psi_{j}\left(\Pi_{i \in P} \Phi_{i}\right) .
$$

In addition to these mass terms arising from $F$ terms, the fields in the flat direction with VEV's set by the FI term will acquire masses from $D$ terms via the super Higgs mechanism. The fields with such effective mass terms will acquire string-scale masses and decouple from the theory.

In addition to the trilinear couplings of the original superpotential, effective renormalizable interactions for the light fields may also be generated via

$$
W \sim \Psi_{i} \Psi_{j} \Psi_{k}\left(\Pi_{i \in P} \Phi_{i}\right) .
$$

The existence of such terms was determined first by identifying the gauge invariant effective bilinear and trilinear terms, then subsequently verifying that such gauge invariant terms survive the string selection rules.

The coupling strengths of the effective trilinear terms generated in this way will generally be suppressed compared to the large Yukawa couplings of the original superpotential, which have coupling strengths $\mathcal{O}(g)$ (with the typical value given by $\sqrt{2} g_{\text {string }}=g \sim 0.8$ for the models considered, as is discussed in Sec. III). In general, the coefficients of the superpotential terms of order $K+3$ are given by

$$
\frac{\alpha_{K+3}}{M_{P l}^{K}}=g_{\text {string }}\left(\sqrt{\frac{8}{\pi}}\right)^{K} \frac{C_{K} I_{K}}{M_{P l}^{K}},
$$

where $C_{K}$ is a coefficient of $\mathcal{O}(1)$ which includes different renormalization factors in the operator product expansion (OPE) of the string vertex operators (including the target space gauge group Clebsch-Gordan coefficients), and $I_{K}$ is a world-sheet integral. $I_{1,2}$ for certain typical couplings have been computed numerically by several authors, with the typical result $I_{1} \sim 70, I_{2} \sim 400$ [20]. The coupling strengths of the effective trilinear terms depend on these coefficients and the values of the VEV's involved, which are set by the (model-dependent) FI term. In this way, these couplings can 
naturally provide a hierarchy, with implications for generating fermion textures in the quark and lepton sectors.

\section{B. Results: Model CHL5}

The model we have chosen as a prototype model to analyze is model 5 of [9]. Prior to vacuum restabilization, the model has the gauge group

$$
\begin{aligned}
& \left\{S U(3)_{C} \times S U(2)_{L}\right\}_{\mathrm{obs}} \times\left\{S U(4)_{2} \times S U(2)_{2}\right\}_{\mathrm{hid}} \\
& \quad \times U(1)_{A} \times U(1)^{6},
\end{aligned}
$$

and a particle content that includes the following chiral superfields in addition to the MSSM fields:

$$
\begin{aligned}
& 6(1,2,1,1)+(3,1,1,1)+(\overline{3}, 1,1,1)+4(1,2,1,2)+2(1,1,4,1) \\
& +10(1,1, \overline{4}, 1)+8(1,1,1,2)+5(1,1,4,2)+(1,1, \overline{4}, 2) \\
& +8(1,1,6,1)+3(1,1,1,3)+42(1,1,1,1)
\end{aligned}
$$

where the representation under $\left(S U(3)_{C}, S U(2)_{L}\right.$, $\left.S U(4)_{2}, S U(2)_{2}\right)$ is indicated. We refer the reader to $[9,1,3]$ for the complete list of fields with their $U(1)$ charges.

The SM hypercharge is determined as a linear combination of the six non-anomalous $U(1)$ 's, subject to the conditions that the MSSM fields have the appropriate quantum numbers, and that the remaining fields can be grouped into mirror pairs under $U(1)_{Y}$ (in the attempt to avoid the presence of strictly massless colored or charged fermion fields in the theory). In this model, these criteria lead to a unique definition of $U(1)_{Y}[9,3]$, with Kač-Moody level $k_{Y}=\frac{11}{3}$ (to be compared with the MSSM value of $k_{Y}=\frac{5}{3}$ ).

We presented a complete list of the $D$ flat directions which can be proved to be $F$ flat to all orders in the superpotential in [1], and written more explicitly in Table II in [3]. In [3], we analyzed the $P_{1} P_{2} P_{3}$ subset of the flat directions, which includes the two representative directions $P_{1}^{\prime} P_{2}^{\prime} P_{3}^{\prime}$ and $\left.P_{2} P_{3}\right|_{F}$. The maximum number of $U(1)^{\prime}$ 's is broken in these flat directions, which leave an additional $U(1)^{\prime}$ as well as $U(1)_{Y}$ unbroken [1]. The unbroken $U(1)^{\prime}$ has $k_{Y^{\prime}}$ $=4167 / 250 \simeq 16.67$. The complete list of fields with their $U(1)_{Y}$ and $U(1)^{\prime}$ charges is presented in Tables $\mathrm{I}(\mathrm{a})-\mathrm{I}(\mathrm{c})$.

The VEV's of the fields in the most general $P_{1} P_{2} P_{3}$ flat direction are of the form

$$
\begin{gathered}
\left|\varphi_{27}\right|^{2}=2 x^{2}, \quad\left|\varphi_{28(29)}\right|^{2}=x^{2}-\left|\psi_{1}\right|^{2}, \\
\left|\varphi_{30}\right|^{2}=\left|\psi_{1}\right|^{2}, \quad\left|\varphi_{4(5)}\right|^{2}=\left|\psi_{2}\right|^{2}, \\
\left|\varphi_{2(3)}\right|^{2}=\left|\psi_{1}\right|^{2}-\left|\psi_{2}\right|^{2}, \quad\left|\varphi_{12(13)}\right|^{2}=\left|\psi_{1}\right|^{2}-\left|\psi_{2}\right|^{2}, \\
\left|\varphi_{10(11)}\right|^{2}=\left|\psi_{2}\right|^{2},
\end{gathered}
$$

$(3,2,1,1)$

$(\overline{3}, 1,1,1)$

$(3,1,1,1)$
$(1,2,1,2)$

$(1,1,4,1)$

$(1,1, \overline{4}, 1)$

$(1,1,1,2)$

$(1,1,4,2)$

$(1,1,1,3)$

TABLE I. (a) List of non-Abelian non-singlet observable sector fields in the model with their charges under hypercharge and $U(1)^{\prime}$. (b) List of non-Abelian non-singlet hidden sector fields in the model with their charges under hypercharge and $U(1)^{\prime}$. (c) List of non-Abelian singlet fields in the model with their charges under hypercharge and $U(1)^{\prime}$.

$\left(S U(3)_{C}, S U(2)_{L}\right.$, $\left.S U(4)_{2}, S U(2)_{2}\right)$

(a)

$6 Q_{Y} \quad 100 Q_{Y^{\prime}}$

$\begin{array}{ccc}Q_{a} & 1 & 68 \\ Q_{b} & 1 & 68 \\ Q_{c} & 1 & -71\end{array}$

$\begin{array}{lll}Q_{c} & 1 & 6 \\ u_{a}^{c} & -4 & 6\end{array}$

$\begin{array}{lll}u_{b}^{c} & -4 & 6\end{array}$

$\begin{array}{lll}u_{c}^{c} & -4 & -133\end{array}$

$\begin{array}{lll}d_{a}^{c} & 2 & -3\end{array}$

$\begin{array}{lll}d_{b}^{c} & 2 & 136\end{array}$

$\begin{array}{lll}d_{c}^{c} & 2 & -3\end{array}$

$\begin{array}{lll}d_{c}^{c} & 2 & -3\end{array}$

$\begin{array}{lll}\bar{h}_{a} & 3 & -74\end{array}$

$\begin{array}{lll}\bar{h}_{b} & 3 & 65\end{array}$

$\begin{array}{lll}\bar{h}_{c} & 3 & 204\end{array}$

$\begin{array}{lll}\bar{h}_{d} & 3 & 65\end{array}$

$\begin{array}{lll}h_{a} & -3 & 74\end{array}$

$h_{b} \quad-3 \quad-65$

$\begin{array}{lll}h_{c} & -3 & -65\end{array}$

$\begin{array}{lll}h_{d} & -3 & -65\end{array}$

$\begin{array}{lll}h_{e} & -3 & -204\end{array}$

$\begin{array}{lll}h_{f} & -3 & -65\end{array}$

$h_{g} \quad-3 \quad-65$

$\begin{array}{lll}\mathcal{D}_{a} & -2 & -136\end{array}$

(b)

\begin{tabular}{ccc}
$D_{1-4}$ & 0 & 0 \\
$F_{1,2}$ & -3 & -65 \\
$\bar{F}_{1,2}$ & 3 & 65 \\
$\bar{F}_{3-6}$ & 3 & 65 \\
$\bar{F}_{7,8}$ & -3 & -65 \\
$\bar{F}_{9,10}$ & -3 & -65 \\
$H_{1,2}$ & 3 & 65 \\
$H_{3,4}$ & 3 & 204 \\
$H_{5,7}$ & -3 & -65 \\
$H_{6,8}$ & -3 & 74 \\
$E_{1,2}$ & 0 & -139 \\
$E_{3}$ & 0 & 0 \\
$E_{4,5}$ & 0 & 0 \\
$\bar{E}_{1}$ & 0 & 0 \\
$S_{1}$ & 0 & 0 \\
$S_{2}$ & 0 & 0 \\
$S_{3}$ & 0 & 0 \\
$S_{4}$ & 0 & 0 \\
$S_{5}$ & 0 & 0 \\
$S_{6,7}$ & 0 & 139 \\
$S_{8}$ & 0 & 0 \\
$T_{1}$ & 0 & 0 \\
$T_{2}$ & 0 & 139 \\
$T_{3}$ & 0 & 0 \\
\hline & &
\end{tabular}


TABLE I. (Continued).

(c)

\begin{tabular}{lccccc} 
& $6 Q_{Y}$ & $100 Q_{Y^{\prime}}$ & & $6 Q_{Y}$ & $100 Q_{Y^{\prime}}$ \\
\hline$e_{a, c}^{c}$ & 6 & -9 & $e_{b}^{c}$ & 6 & -9 \\
$e_{d, g}^{c}$ & 6 & 130 & $e_{e}^{c}$ & 6 & 130 \\
$e_{f}^{c}$ & 6 & 130 & $e_{h}^{c}$ & 6 & 130 \\
$e_{i}^{c}$ & 6 & -9 & $e_{a, b}$ & 6 & -130 \\
$e_{c}$ & 6 & -130 & $e_{d, e}$ & -6 & 9 \\
$e_{f}$ & -6 & -269 & & & \\
& & & & & \\
$\varphi_{1}$ & 0 & 0 & $\varphi_{2,3}$ & 0 & 0 \\
$\varphi_{4,5}$ & 0 & 0 & $\varphi_{6,7}$ & 0 & 0 \\
$\varphi_{8,9}$ & 0 & 0 & $\varphi_{10,11}$ & 0 & 0 \\
$\varphi_{12,13}$ & 0 & 0 & $\varphi_{14,15}$ & 0 & 0 \\
$\varphi_{16}$ & 0 & 0 & $\varphi_{17}$ & 0 & 0 \\
$\varphi_{18,19}$ & 0 & -139 & $\varphi_{20,21}$ & 0 & -139 \\
$\varphi_{22}$ & 0 & -139 & $\varphi_{23}$ & 0 & 0 \\
$\varphi_{24}$ & 0 & 0 & $\varphi_{25}$ & 0 & 139 \\
$\varphi_{26}$ & 0 & 0 & $\varphi_{27}$ & 0 & 0 \\
$\varphi_{28,29}$ & 0 & 0 & $\varphi_{30}$ & 0 & 0 \\
\hline \hline
\end{tabular}

with

$$
x=\frac{\sqrt{|\xi|}}{8}=0.013 M_{\mathrm{Pl}} \text {, }
$$

and $\left|\psi_{1,2}\right|$ are free VEV's of the moduli space, subject to the restrictions that $x^{2} \geqslant\left|\psi_{1}\right|^{2} \geqslant\left|\psi_{2}\right|^{2}$.

The first representative flat direction $P_{1}^{\prime} P_{2}^{\prime} P_{3}^{\prime}$ has VEV's given by the general case (7), with no further restrictions on $\left|\psi_{1,2}\right|$. However, the second representative flat direction $\left.P_{2} P_{3}\right|_{F}$ corresponds to the case in which constraints must be imposed on the free VEV's in such a way that the contributions from different $F$ terms cancel and $\left|\varphi_{28(29)}\right|^{2}=0$; these constraints are $x^{2}=\left|\psi_{1}\right|^{2}=2\left|\psi_{2}\right|^{2}$, and $\pi$ phase difference between the combination of VEV's $\varphi_{4(5)} \varphi_{10(11)}$ and $\varphi_{2(3)} \varphi_{12(13)}[1]$.

For each of these flat directions, we computed the effective mass terms and determined the mass eigenstates in [3]. In addition to the fields which become massive from these couplings, it can be shown that of the fields in each flat direction, five of the associated chiral superfields become massive due to the super Higgs mechanism. In the $P_{1}^{\prime} P_{2}^{\prime} P_{3}^{\prime}$ flat direction, two of the chiral superfields remain massless (moduli), but they do not couple to the rest of the fields at the level of the effective trilinear terms. In the $\left.P_{2} P_{3}\right|_{F}$ flat direction, the remaining complex field gets a mass of order [Yukawa] $\times$ [field $\mathrm{VEV}]$ due to cancellations of $F$ term contributions and forms, along with its superpartner, a massive chiral superfield. In this case, the fields with zero VEV's which couple linearly in these terms also acquire masses of the same order.

The effective trilinear couplings along each of the representative flat directions were also determined in [3]. In this model, the numerical analysis (see also [11]) of the string amplitudes yields, along with the $x$ as the typical VEV of the
TABLE II. List of massless states (excluding the two moduli) for the $P_{1}^{\prime} P_{2}^{\prime} P_{3}^{\prime}$ flat direction [3], where $N_{1} \equiv 2 g^{2} x^{2}$ $+\left(\alpha_{4}^{(1)}\left|\psi_{2}\right|^{2} / M_{P l}\right)^{2}$ with the VEV parameters $x, \psi_{1}$ and $\psi_{2}$ defined in Eqs. (7).

\begin{tabular}{ll}
\hline \hline & Massless fields \\
\hline & $Q_{a}, Q_{b}, Q_{c}$ \\
& $u_{a}^{c}, u_{b}^{c}, u_{c}^{c}$ \\
& $d_{a}^{c}, d_{b}^{c}, d_{c}^{c}, d_{d}^{c}, \mathcal{D}_{a}$ \\
& $\bar{h}_{a}, \bar{h}_{c}$ \\
& $h_{a}, h_{c}, h_{d}, h_{e}$ \\
& $h_{b}^{\prime}=\frac{1}{\sqrt{N_{1}}}\left[-\frac{\alpha_{4}^{(1)}\left|\psi_{2}\right|^{2}+\alpha_{4}^{(2)}\left(\left|\psi_{1}\right|^{2}-\left|\psi_{2}\right|^{2}\right)}{M_{P l}} h_{f}+\sqrt{2} g x h_{b}\right]$ \\
& $e_{a}^{c}, e_{b}^{c}, e_{c}^{c}, e_{e}^{c}, e_{f}^{c}, e_{h}^{c}, e_{i}^{c}$ \\
& $e_{c}, e_{d}, e_{e}, e_{f}$ \\
& $\varphi_{3}, \varphi_{11}, \varphi_{18}, \varphi_{19}, \varphi_{20}, \varphi_{22}, \varphi_{24}, \varphi_{28}$ \\
& $\varphi_{12}^{\prime}=\frac{1}{\left|\psi_{1}\right|}\left(-\sqrt{\left|\psi_{1}\right|^{2}-\left|\psi_{2}\right|^{2}} \varphi_{12}+\left|\psi_{2}\right| \varphi_{4}\right)$ \\
& $D_{1}, D_{2}, D_{3}, D_{4}$ \\
& $\bar{F}_{3}, \bar{F}_{4}, \bar{F}_{5}, \bar{F}_{6}, \bar{F}_{7}, \bar{F}_{8}, \bar{F}_{9}, \bar{F}_{10}$ \\
& $H_{1}, H_{2}, H_{3}, H_{4}, H_{5}, H_{6}, H_{7}, H_{8}$ \\
& $E_{1}, E_{2}, E_{3}, E_{4}, E_{5}, \bar{E}_{1}$ \\
& $S_{2}, S_{4}, S_{6}, S_{7}, S_{8}$ \\
& $S_{1}^{\prime}=\frac{1}{\left|\psi_{1}\right|}\left(-\sqrt{\left|\psi_{1}\right|^{2}-\left|\psi_{2}\right|^{2}} S_{3}+\left|\psi_{2}\right| S_{1}\right)$ \\
& $T_{1}, T_{2}, T_{3}$ \\
\hline \hline &
\end{tabular}

fields along the flat direction, the effective Yukawa coupling at the fourth order $\sim 0.8$, while the fifth order terms have strengths $\sim 0.1$ (using the typical values of $I_{1} \sim 70$ and $I_{2}$ $\sim 400$ [20]). Therefore, in this model the effective trilinear couplings arising from fourth order terms are competitive in strength to the elementary trilinear terms, while the higher order contributions are indeed suppressed [11]. Of course, the precise values for each term will depend on the particular fields involved. In addition, the coupling strengths can depend on the undetermined VEV's in the $P_{1}^{\prime} P_{2}^{\prime} P_{3}^{\prime}$ flat direction.

\section{III. $P_{1}^{\prime} P_{2}^{\prime} P_{3}^{\prime}$ FLAT DIRECTION}

\section{A. Effective superpotential}

The $P_{1}^{\prime} P_{2}^{\prime} P_{3}^{\prime}$ direction involves the set of fields $\left\{\varphi_{2}, \varphi_{5}, \varphi_{10}, \varphi_{13}, \varphi_{27}, \varphi_{29}, \varphi_{30}\right\}$. The VEV's correspond to the most general case given in Eqs. (7), such that they depend on two free (but bounded) parameters.

It is straightforward to determine the mass eigenstates, which were calculated in [3]. In Table II we list the surviving massless states. These states include both the usual MSSM states and related exotic [non-chiral under $S U(2)_{L}$ ] states, such as a fourth $\left[S U(2)_{L}\right.$ singlet $]$ down-type quark, extra fields with the same quantum numbers as the lepton singlet superfields, and extra Higgs doublets. There are other massless states with exotic quantum numbers (including fractional electric charge) and states which are non-Abelian represen- 
tations under both the hidden and observable sector gauge groups and thus directly mix the two sectors. As previously discussed, there are two additional massless states (moduli) associated with fields which appear in the flat direction but which do not have fixed VEV's (but do not couple to other fields at the level of an effective trilinear term) and are not listed in Table II.

The effective trilinear couplings involving the observable sector fields assume the form [3]

$$
\begin{aligned}
W_{3}= & g Q_{c} u_{c}^{c} \bar{h}_{c}+g Q_{c} d_{b}^{c} h_{c}+\frac{\alpha_{4}^{(4)}}{M_{P l}} \sqrt{1-\lambda_{1}^{2}} Q_{c} d_{d}^{c} h_{a} \\
& +\frac{g}{\sqrt{2}} e_{a}^{c} h_{a} h_{c}+\frac{g}{\sqrt{2}} e_{f}^{c} h_{d} h_{c}+\frac{\sqrt{2} \alpha_{5}^{(1)} x^{2}}{M_{P l}^{2}} \lambda_{2} e_{h}^{c} h_{e} h_{a} \\
& +\frac{\sqrt{2} \alpha_{5}^{(2)} x^{2}}{M_{P l}^{2}} \sqrt{\lambda_{1}^{2}-\lambda_{2}^{2}} e_{e}^{c} h_{e} h_{a}+g \bar{h}_{c} h_{b}^{\prime} \varphi_{20}^{\prime},
\end{aligned}
$$

in which

$$
\lambda_{2} \equiv \frac{\left|\psi_{2}\right|}{x} \leqslant \lambda_{1} \equiv \frac{\left|\psi_{1}\right|}{x} \leqslant 1
$$

are free parameters and $\varphi_{20}^{\prime}=\left(1 / \sqrt{1+r^{2}}\right)\left(\varphi_{20}-r \varphi_{22}\right)$, with $r \equiv\left[\alpha_{4}^{(1)} \lambda_{2}^{2}+\alpha_{4}^{(2)}\left(\lambda_{1}^{2}-\lambda_{2}^{2}\right)\right] x /\left(\sqrt{2} g M_{P l}\right)$.

The superpotential implies generic features independent of the details of the soft supersymmetry breaking, which have been analyzed in [3]: (i) With the identification of the fields $\bar{h}_{c}$ and $h_{c}$ with the standard electroweak Higgs doublets, the Yukawa couplings indicate $t-b$ and $\tau$ - $\mu$ Yukawa unification with equal string scale Yukawa couplings $g$ and $g / \sqrt{2}$, respectively; (ii) there is no elementary or effective canonical $\mu$ term; (iii) there is a possibility of lepton-number violating couplings and thus no stable LSP. In particular, we identify the fields $\left\{h_{e}, h_{a}, h_{d}\right\}$ as the lepton doublets, and hence the couplings $Q_{c} d_{d}^{c} h_{a}$ and $e_{e, h}^{c} h_{a} h_{e}$ violate lepton number. The fields $h_{b}^{\prime}$ and $\varphi_{20}^{\prime}$ can play the role of additional Higgs fields. $\bar{h}_{a}$ has the quantum numbers of a Higgs doublet, but does not enter $W_{3}$, and hence there is no mechanism for it to develop a VEV.

\section{B. Symmetry breaking patterns}

To address the gauge symmetry breaking scenarios for this model, we introduce soft supersymmetry breaking mass parameters and run the renormalization group equations (RGE's) from the string scale to the electroweak scale. While the qualitative features of the analysis are independent of the details of the soft breaking, we choose to illustrate the analysis with a specific example with a realistic $Z-Z^{\prime}$ hierarchy.

We wish to investigate the $U(1)^{\prime}$ symmetry breaking scenarios discussed in $[13,14]$, which indicate that in the class of string models considered, the $U(1)^{\prime}$ symmetry breaking is either at the electroweak $(\mathrm{TeV})$ scale or at an intermediate scale (if the symmetry breaking takes place along a $D$ flat direction). An inspection of the massless spectrum in Table
$\mathrm{I}(\mathrm{c})$ indicates that the singlet field $\varphi_{25}$ is required for a $D$ flat direction [and hence the intermediate scale $U(1)^{\prime}$ symmetry breaking scenario]; however, this field acquires a string-scale mass for this direction, and decouples from the theory. We conclude that in this case, an intermediate scale breaking scenario is not possible, and hence the breaking of the $U(1)^{\prime}$ is necessarily at the electroweak scale.

Though hidden sector non-Abelian fields are not directly or indirectly coupled to the observable sector non-Abelian fields at the trilinear order in the superpotential, the $U(1)^{\prime}$ could be radiatively broken along with some of the hidden non-Abelian groups, and in such cases the breaking of the hidden sector is connected with the $S U(2) \times U(1)_{Y}$ symmetry breaking in the observable sector through $U(1)^{\prime}$.

As discussed in [13,14], several scenarios exist which can lead to the possibility of a realistic $Z-Z^{\prime}$ hierarchy. The scenario in which only the two MSSM Higgs fields $h_{c}, \bar{h}_{c}$ acquire VEV's breaks both $U(1)_{Y}$ and $U(1)^{\prime}$, but leads to a light $Z^{\prime}$ with $M_{Z^{\prime}} \sim \mathcal{O}\left(M_{Z}\right)$, which is already excluded by experiments.

To have a realistic $Z-Z^{\prime}$ hierarchy, we require that a SM singlet field that is charged under the $U(1)^{\prime}$ acquire a VEV. However, since the canonical $\mu$ term that couples both $h_{c}$ and $\bar{h}_{c}$ to a SM singlet is absent, and instead there is a noncanonical $\mu$ term $\bar{h}_{c} h_{b}^{\prime} \varphi_{20}^{\prime}$, the minimization of the potential requires that the additional Higgs doublet $h_{b}^{\prime}$ acquire a nonzero VEV. Therefore, we consider the most general case in which $h_{b}^{\prime}$ and $\varphi_{20}^{\prime} \equiv s$ acquire VEV's in addition to $\bar{h}_{c}$ and $h_{c}$. After adding the required soft supersymmetry breaking terms, the potential is given by

$$
V=V_{F}+V_{D}+V_{\text {soft }},
$$

with

$$
\begin{gathered}
V_{F}=\Gamma_{s}^{2}|s|^{2}\left(\left|\bar{h}_{c}\right|^{2}+\left|h_{b}^{\prime}\right|^{2}\right)+\Gamma_{s}^{2}\left|\bar{h}_{c} \cdot h_{b}^{\prime}\right|^{2} \\
V_{D}=\frac{G^{2}}{8}\left(\left|\bar{h}_{c}\right|^{2}-\left|h_{c}\right|^{2}-\left|h_{b}^{\prime}\right|^{2}\right)^{2}+\frac{g_{2}^{2}}{2}\left[\left|\bar{h}_{c}^{*} h_{b}^{\prime}\right|^{2}+\left|\bar{h}_{c}^{*} h_{c}\right|^{2}\right. \\
\left.+\left|h_{c}^{*} h_{b}^{\prime}\right|^{2}\right]-\frac{g_{2}^{2}}{2}\left|h_{b}^{\prime}\right|^{2}\left|h_{c}\right|^{2}+\frac{g_{1}^{\prime 2}}{2}\left(Q_{1}\left|h_{c}\right|^{2}+Q_{2}\left|\bar{h}_{c}\right|^{2}\right. \\
\left.+Q_{3}\left|h_{b}^{\prime}\right|^{2}+Q_{s}|s|^{2}\right)^{2} \\
V_{\text {soft }}=m_{\bar{h}_{c}}^{2}\left|\bar{h}_{c}\right|^{2}+m_{h_{c}}^{2}\left|h_{c}\right|^{2}+m_{h_{b}^{\prime}}^{2}\left|h_{b}^{\prime}\right|^{2}+m_{s}^{2}|s|^{2} \\
-\left(A \Gamma_{s} \bar{h}_{c} \cdot h_{b}^{\prime} s+\text { H.c. }\right)
\end{gathered}
$$

in which $\Gamma_{s}$ is the coefficient for the coupling $\bar{h}_{c} h_{b}^{\prime} \varphi_{20}^{\prime}, G^{2}$ $=g_{Y}^{2}+g_{2}^{2}$ (with $g_{Y}^{2}=\frac{3}{11} g_{1}^{2}$ ), and

$$
h_{c}=\left(\begin{array}{c}
h_{c}^{0} \\
h_{c}^{-}
\end{array}\right), \quad \bar{h}_{c}=\left(\begin{array}{c}
\bar{h}_{c}^{+} \\
\bar{h}_{c}^{0}
\end{array}\right), \quad h_{b}^{\prime}=\left(\begin{array}{c}
h_{b}^{\prime 0} \\
h_{b}^{\prime-}
\end{array}\right) .
$$

The $U(1)^{\prime}$ charges of $\left\{h_{c}, \bar{h}_{c}, h_{b}^{\prime}, s\right\}$ are denoted by $Q_{1}, Q_{2}, Q_{3}$, and $Q_{s}$, respectively. We can take $A \Gamma_{s}$ real and 
positive without loss of generality by an appropriate choice of the global phases of the fields. By a suitable gauge rotation we also take $\left\langle\bar{h}_{c}^{0}\right\rangle$ and $\langle s\rangle$ real and positive, which implies that $\left\langle h_{b}^{\prime 0}\right\rangle$ is real and positive at the minimum. However, the phase of the $h_{c}$ field is not determined, due to the absence of an effective $\mu$ term involving $h_{c}$ in Eq. (11). This additional global $U(1)$ symmetry leads to the presence of a Goldstone boson in the massless spectrum, as discussed below. For notational simplicity, we define $\sqrt{2}\left\langle h_{c}^{0}\right\rangle \equiv v_{1}$, $\sqrt{2}\left\langle\bar{h}_{c}^{0}\right\rangle \equiv v_{2}, \sqrt{2}\left\langle h_{b}^{\prime 0}\right\rangle \equiv v_{3}$, and $\sqrt{2}\langle s\rangle \equiv s$.

The $Z-Z^{\prime}$ mass matrix is given by

$$
\left(M^{2}\right)_{Z-Z^{\prime}}=\left(\begin{array}{cc}
M_{Z}^{2} & \Delta^{2} \\
\Delta^{2} & M_{Z^{\prime}}^{2}
\end{array}\right)
$$

where

$$
\begin{aligned}
M_{Z}^{2} & =\frac{1}{4} G^{2}\left(v_{1}^{2}+v_{2}^{2}+v_{3}^{2}\right) \\
M_{Z^{\prime}}^{2} & =g_{1}^{\prime 2}\left(v_{1}^{2} Q_{1}^{2}+v_{2}^{2} Q_{2}^{2}+v_{3}^{2} Q_{3}^{2}+s^{2} Q_{S}^{2}\right) \\
\Delta^{2} & =\frac{1}{2} g_{1}^{\prime} G\left(v_{1}^{2} Q_{1}+v_{3}^{2} Q_{3}-v_{2}^{2} Q_{2}\right)
\end{aligned}
$$

with mass eigenvalues

$$
M_{Z_{1}, Z_{2}}^{2}=\frac{1}{2}\left[M_{Z}^{2}+M_{Z^{\prime}}^{2} \mp \sqrt{\left(M_{Z}^{2}-M_{Z^{\prime}}^{2}\right)^{2}+4 \Delta^{4}}\right] .
$$

The $Z-Z^{\prime}$ mixing angle $\alpha_{Z-Z^{\prime}}$ is given by

$$
\alpha_{Z-Z^{\prime}}=\frac{1}{2} \arctan \left(\frac{2 \Delta^{2}}{M_{Z^{\prime}}^{2}-M_{Z}^{2}}\right),
$$

which is constrained to be less than a few times $10^{-3}$.

The only possibility [3] for a realistic hierarchy is for the symmetry breaking to be characterized by a large $[\mathcal{O}(\mathrm{TeV})]$ value for the SM singlet VEV $s$, with the $S U(2)_{L} \times U(1)_{Y}$ breaking at a lower scale due to accidental cancellations.

We now proceed with the analysis of the renormalization group equations.

\section{Running of the gauge couplings}

As discussed in [3], we determine the gauge coupling constant $g=0.80$ at the string scale by assuming $\alpha_{s}=0.12$ (experimental value) at the electroweak scale and evolving $g_{3}$ to the string scale. We find that $g=0.80$ is slightly higher than that of the MSSM, due to the presence of one additional vectorlike exotic quark pair. The electroweak scale values of the other gauge couplings are determined by their (1-loop) RGE's, taking $g=0.8$ at the string scale as an input. The running of the gauge couplings is presented in Fig. 1, and the $\beta$ functions are listed in Table III, including the Kač-Moody levels for the $U(1)$ gauge factors $\left(k_{Y}=11 / 3, k^{\prime}=16.67\right)$ and $k=2$ for the hidden sector non-Abelian groups.

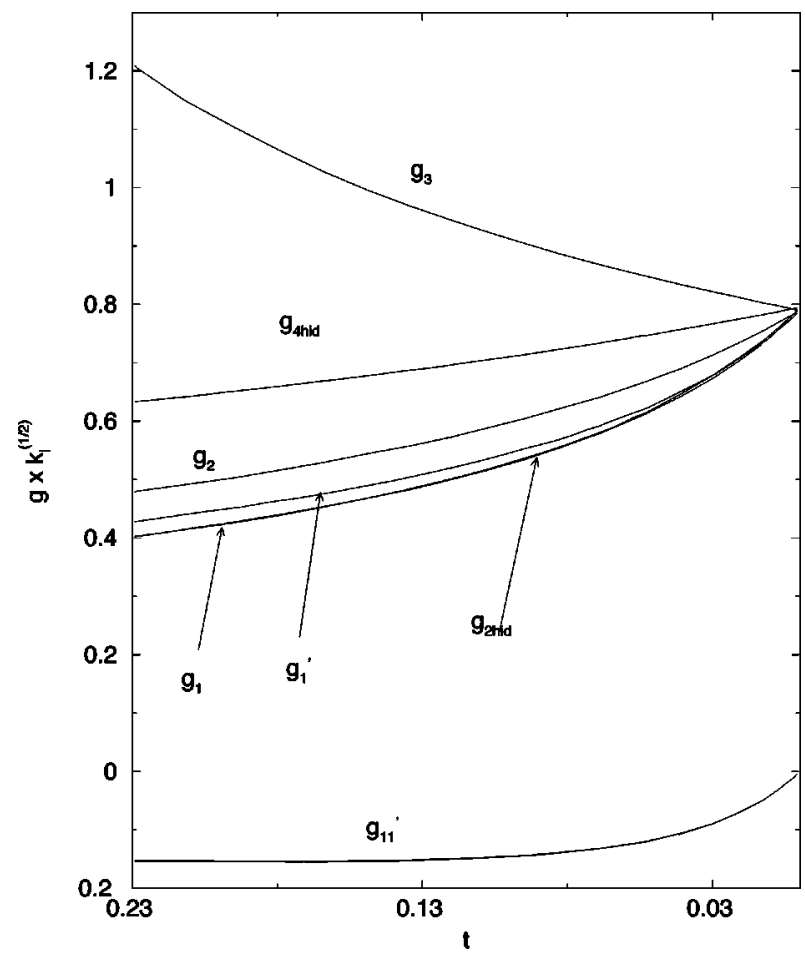

FIG. 1. Variation of the gauge couplings $\times \sqrt{k}$ with the scale for the $P_{1}^{\prime} P_{2}^{\prime} P_{3}^{\prime}$ flat direction, with $t=\left(1 / 16 \pi^{2}\right) \ln \left(\mu / M_{\text {String }}\right), M_{\text {String }}$ $=5 \times 10^{17} \mathrm{GeV}$, and $g\left(M_{\text {String }}\right)=0.80$. The couplings include the factor $\sqrt{k}$, where $\mathrm{k}$ corresponds to the associated Kač-Moody level (see the caption of Table III for the values of $k$ ).

The low energy values of the gauge couplings are not correct due to the exotic matter and non-standard $k_{Y}$. Surprisingly, $\sin ^{2} \theta_{W} \sim 0.16$ is not too different from the experimental value 0.23 , and $g_{2}=0.48$ is to be compared to the experimental value 0.65 . As a result of a large number of massless non-Abelian fields in the hidden sector, the hidden sector gauge couplings are not asymptotically free. Therefore, dynamical supersymmetry breaking due to strong coupling dynamics in the hidden sector is not possible in this model.

\section{Running of the Yukawa couplings}

The values of the Yukawa couplings at the string scale after vacuum restabilization are indicated in Eq. (9). There

TABLE III. Effective beta-functions for the two representative flat directions, defined as $\beta_{i} \equiv \beta_{i}^{0} / k_{i}$, where $\beta_{i}^{0}$ and $k_{i}$ are the betafunction and the Kač-Moody level for a particular gauge group factor, respectively. The subscripts $1,2,3,1^{\prime}, 2 \mathrm{hid}$, 4 hid refer to $U(1)_{Y}, S U(2)_{L}, S U(3)_{C}, U(1)^{\prime}, S U(2)_{2}, S U(4)_{2}$ gauge group factors and $11^{\prime}$ refers to the $U(1)_{Y}$ and $U(1)^{\prime}$ knetic mixing. The Kač-Moody levels are $k_{1}=11 / 3, k_{2}=k_{3}=1, k_{1}, \simeq 16.67$, and $k_{2 h i d}=k_{4 h i d}=2$.

\begin{tabular}{lccccccc}
\hline \hline Effective $\beta$ & $\beta_{1}$ & $\beta_{2}$ & $\beta_{3}$ & $\beta_{1}$, & $\beta_{11^{\prime}}$ & $\beta_{2 h i d}$ & $\beta_{4 h i d}$ \\
\hline$P_{1}^{\prime} P_{2}^{\prime} P_{3}^{\prime}$ flat direction & 10.0 & 6.0 & -2.0 & 10.2 & 4.8 & 10.0 & 2.0 \\
$\left.P_{2} P_{3}\right|_{F}$ flat direction & 10.3 & 7.0 & -2.0 & 10.6 & 5.0 & 10.0 & 3.0 \\
\hline \hline
\end{tabular}


TABLE IV. $P_{1}^{\prime} P_{2}^{\prime} P_{3}^{\prime}$ flat direction: values of the parameters at $M_{\text {String }}$ and $M_{Z}$, with $M_{Z^{\prime}}=735 \mathrm{GeV}$ and $\alpha_{Z-Z^{\prime}}=0.005$. All mass parameters are given in $\mathrm{GeV}$. Other soft supersymmetry breaking mass parameters approximately decouple, and are not presented.

\begin{tabular}{|c|c|c|c|c|c|}
\hline & $M_{Z}$ & $M_{\text {String }}$ & & $M_{Z}$ & $M_{\text {String }}$ \\
\hline$g_{1}$ & 0.41 & 0.80 & $M_{1}$ & 444 & 1695 \\
\hline$g_{2}$ & 0.48 & 0.80 & $M_{2}$ & 619 & 1695 \\
\hline$g_{3}$ & 1.23 & 0.80 & $M_{3}$ & 4040 & 1695 \\
\hline$g_{1}^{\prime}$ & 0.43 & 0.80 & $M_{1}^{\prime}$ & 392 & 1695 \\
\hline$\Gamma_{Q 1}$ & 0.96 & 0.80 & $A_{Q 1}$ & 3664 & 8682 \\
\hline$\Gamma_{Q 2}$ & 0.93 & 0.80 & $A_{Q 2}$ & 4070 & 9000 \\
\hline$\gamma_{Q 3}$ & 0.27 & 0.08 & $A_{Q 3}$ & 5018 & 1837 \\
\hline$\Gamma_{l 1}$ & 0.30 & 0.56 & $A_{l 1}$ & -946 & 4703 \\
\hline$\Gamma_{l 2}$ & 0.36 & 0.56 & $A_{l 2}$ & -707 & 4532 \\
\hline$\Gamma_{l 3}$ & 0.06 & 0.05 & $A_{l 3}$ & 4613 & 4425 \\
\hline$\Gamma_{l 4}$ & 0.11 & 0.13 & $A_{l 4}$ & 4590 & 4481 \\
\hline$\Gamma_{s}$ & 0.22 & 0.80 & $A$ & 1695 & 12544 \\
\hline$m_{O}^{2}$ & $(2706)^{2}$ & $(2450)^{2}$ & $m_{d}^{2}$ & $(4693)^{2}$ & $(2125)^{2}$ \\
\hline$m_{u}^{2^{c}}$ & $(2649)^{2}$ & $(2418)^{2}$ & $m_{d}^{2_{d}^{d}}$ & $(2734)^{2}$ & $(2486)^{2}$ \\
\hline$m_{\bar{h}}^{2^{c}}$ & $(1008)^{2}$ & $(5622)^{2}$ & $m_{h^{\prime}}^{2^{c}}$ & $(826)^{2}$ & $(2595)^{2}$ \\
\hline$m_{\varphi_{20^{\prime}}}^{n^{c}}$ & $-(518)^{2}$ & $(6890)^{2}$ & $m_{\varphi_{22^{\prime}} 2^{n} b}$ & $(3031)^{2}$ & $(11540)^{2}$ \\
\hline$m_{h}^{2}$ & $(3626)^{2}$ & $(3982)^{2}$ & $m_{h}^{2}$ & $-(224)^{2}$ & $(5633)^{2}$ \\
\hline$m_{h}^{2^{a}}$ & $(3666)^{2}$ & $(4100)^{2}$ & $m_{h}^{n_{h}^{c}}$ & $(4274)^{2}$ & $(4246)^{2}$ \\
\hline$m_{e}^{2^{d}}$ & $(2770)^{2}$ & $(3564)^{2}$ & $m_{e}^{2^{e}}$ & $(2780)^{2}$ & $(3958)^{2}$ \\
\hline$m_{e_{e}}^{e^{a}}$ & $(4195)^{2}$ & $(4254)^{2}$ & $m_{e_{h}}^{2_{f}^{f}}$ & $(4259)^{2}$ & $(4236)^{2}$ \\
\hline
\end{tabular}

are two free parameters $\lambda_{1}$ and $\lambda_{2}$ satisfying the constraints (10). To minimize the effects of the lepton number violating effective Yukawa coupling $Q_{c} d_{d}^{c} h_{a}$, we choose $\lambda_{1}=0.9$ at the string scale. The dependence of $\lambda_{2}$ is all from higher order effective terms, which are numerically supressed and less important. For the sake of definiteness, we take $\lambda_{2}$ $=0.4$. As discussed in Sec. II, we choose typical values for $I_{1,2}$ such that $\alpha_{4} x / M_{P l} \sim 0.8, \alpha_{5} x^{2} / M_{P l}^{2} \sim 0.1$, and an estimation for $I_{3}$ such that $\alpha_{6} x^{3} / M_{P l}^{3} \sim 0.01$. With the choice of $g$ $=0.8$ at the string scale, the initial values of Yukawa couplings are listed in Table IV. In Fig. 2 we present the evolution of the Yukawa couplings with the scale. We denote the Yukawa couplings of the quark doublets by $\Gamma_{Q i}$, the couplings of the lepton doublets by $\Gamma_{l i}$, the coupling of the two Higgs doublets and the singlet by $\Gamma_{s}$ [the numbering follows the order of the terms in Eq. (9)]. Additional Yukawa couplings of non-Abelian singlet fields to hidden sector fields, not displayed in Eq. (9), are listed in Eq. (20) of [3]. Their effects are included in the calculation of the running Yukawas and soft parameters.

The low energy values of the Yukawa couplings can be read off from Table IV. The values of $\Gamma_{Q 1} \sim 0.96$ and $\Gamma_{Q 2}$ $\sim 0.93$ indicate that the $t-b$ degeneracy is mainly broken by $\tan \beta$ at the electroweak scale. The result $\Gamma_{l 1} \sim 0.30, \Gamma_{l 2}$ $\sim 0.36$ indicates that the $\tau-\mu$ degeneracy is only slightly broken at low energy, and $m_{b} / m_{\tau} \sim 2.6$. The value of the Yukawa coupling $\bar{h}_{c} h_{b}^{\prime} \varphi_{20}^{\prime}$, which plays a significant role in the gauge symmetry breaking (in this scenario for which all

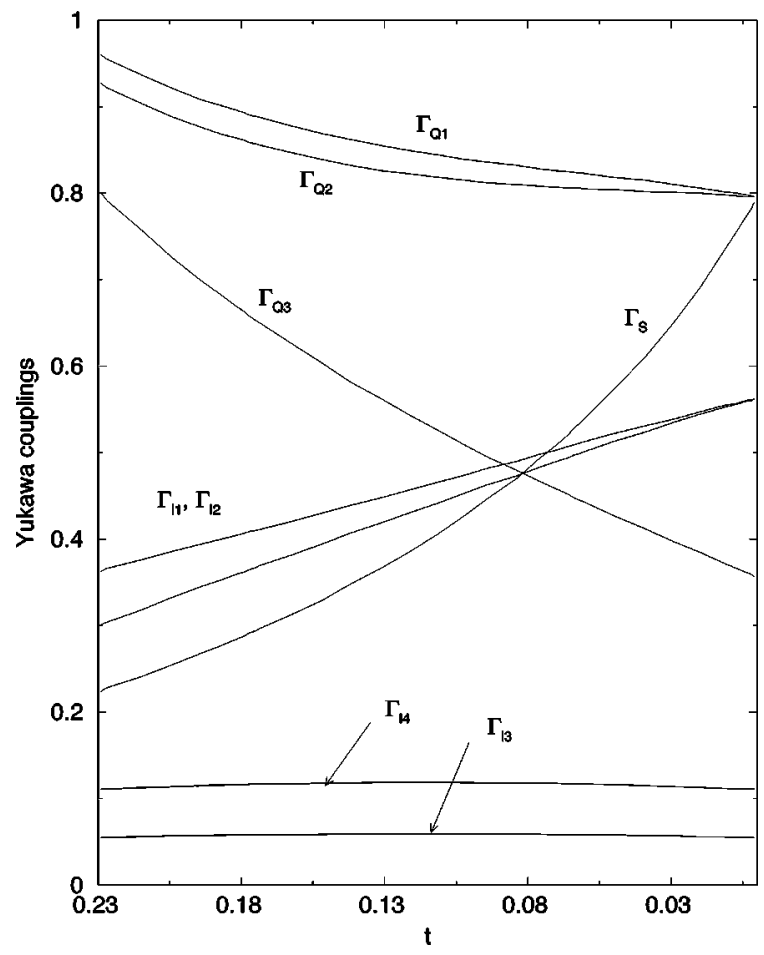

FIG. 2. Running of the Yukawa couplings for the $P_{1}^{\prime} P_{2}^{\prime} P_{3}^{\prime}$ direction. The two free parameters $\lambda_{1}$ and $\lambda_{2}$ are chosen to be 0.9 and 0.4 , respectively.

three fields have non-zero VEV's), is given by $\Gamma_{s}\left(M_{Z}\right)$ $\sim 0.22$.

\section{Running of the soft mass parameters}

We choose to normalize the soft breaking scale by ensuring the correct value of $M_{Z}$ (before mixing with $Z^{\prime}$ ), rather than using $v$ or $M_{W}$; since in this model the gauge couplings $g_{2}$ and $g_{1}$ at $M_{Z}$ have values different from their experimentally observed ones, it implies that $v$ and $M_{W}$ will also differ from their experimentally measured values. With the (incorrect) values $g_{2}\left(M_{Z}\right) \sim 0.48, g_{1}\left(M_{Z}\right) \sim 0.41$ for this model, $v \sim 348 \mathrm{GeV}$ and $M_{W} \sim 82.8 \mathrm{GeV}$ (to be compared with the experimental values $246 \mathrm{GeV}$ and $80.3 \mathrm{GeV}$, respectively).

We find that with universal boundary conditions for the soft supersymmetry breaking mass terms at the string scale, the realistic scenario described above cannot be achieved. In particular, the mass square of the appropriate singlet field $\varphi_{20}^{\prime}$ does not run to negative values; so $\varphi_{20^{\prime}}$ does not acquire a VEV. However, for mild tuning of the boundary conditions it is possible to obtain scenarios in which the $Z^{\prime}$ mass is large enough and the mixing angle sufficiently suppressed to satisfy phenomenological bounds.

We present the initial conditions and low energy values for the soft breaking parameters for an example of such a scenario in Table IV, with $M_{Z^{\prime}}=735 \mathrm{GeV}$, and $\theta_{Z-Z^{\prime}}$ $=0.005$. The running scalar mass squares corresponding to these initial conditions and to the gauge and Yukawa couplings in Figs. 1 and 2 are displayed in Fig. 3.

We see that the mass squares of $h_{c}$ and $\varphi_{20}^{\prime}$ are driven negative at low energy, while the $\bar{h}_{c}$ and $h_{b^{\prime}}$ mass squares 


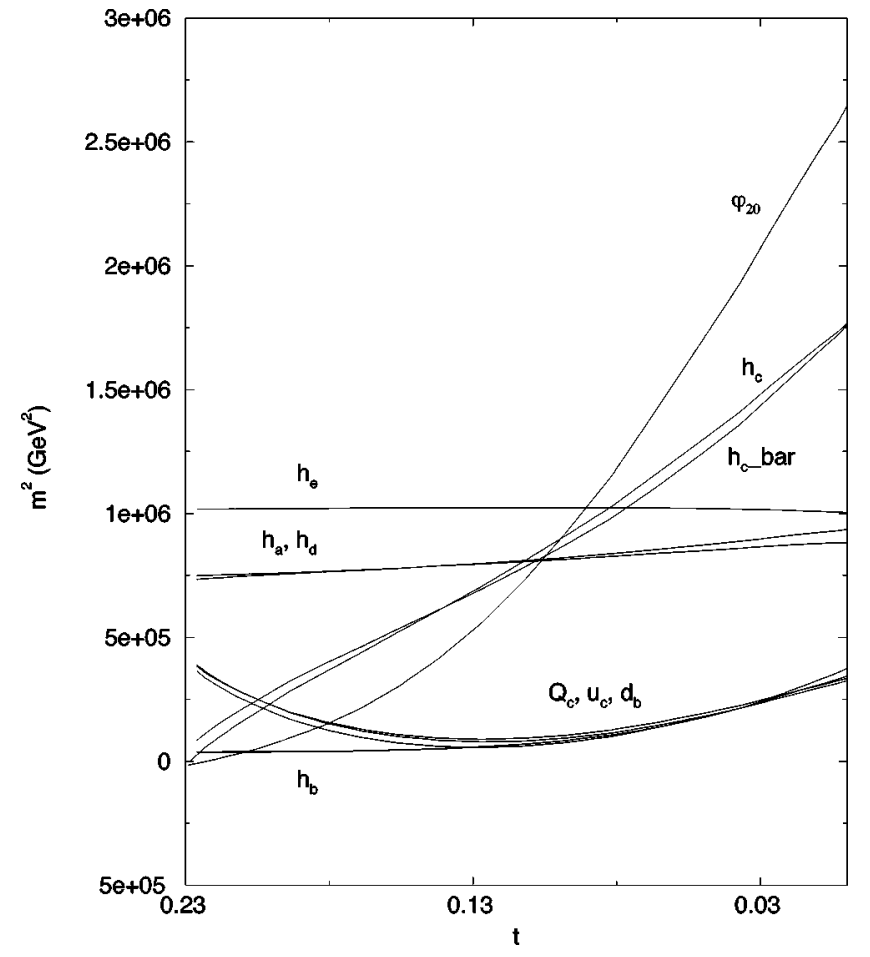

FIG. 3. Running of the soft mass-squared parameters for $P_{1}^{\prime} P_{2}^{\prime} P_{3}^{\prime}$ direction. The non-universal initial conditions are chosen to yield a realistic example for low energy symmetry breaking.

remain positive. Minimization of the potential requires all four of these fields to acquire VEV's, which take the values $\left\langle h_{c}^{0}\right\rangle=90 \mathrm{GeV},\left\langle\bar{h}_{c}^{0}\right\rangle=163 \mathrm{GeV},\left\langle h_{b}^{\prime 0}\right\rangle=161 \mathrm{GeV}$, and $\left\langle\varphi_{20}^{\prime}\right\rangle=3560 \mathrm{GeV}$.

The small values of the doublet VEV's compared to $\left\langle\varphi_{20}^{\prime}\right\rangle$ involve a degree of fine-tuning. From Table IV it is apparent that the typical scale of all soft parameters, and therefore of the VEV's and $Z$ and $Z^{\prime}$ masses, is several TeV. The much smaller values of $\left\langle\bar{h}_{c}^{0}\right\rangle$ and $\left\langle h_{b}^{\prime 0}\right\rangle$ come about because the point $\left\langle\bar{h}_{c}^{0}\right\rangle=\left\langle h_{b}^{\prime 0}\right\rangle=0$ of the potential is a saddle point, with large positive curvature in one direction and a small negative curvature (caused by a near cancellation between the large positive $m_{\bar{h}_{c}}^{2}$ and $m_{h_{b}^{\prime}}^{2}$ terms with the slightly larger negative $A$ term) in the other direction. The small $\left\langle h_{c}^{0}\right\rangle$ is due to the small negative $m_{h_{c}}^{2}$ and the absence of a trilinear term involving $h_{c}$.

\section{Mass spectrum}

We now address the mass spectrum of the model associated with this particular low-energy solution. The large singlet VEV scenario was explored for models with two Higgs doublets and a singlet connected by a canonical $\mu$ term in [14]. In the present model, there are three Higgs doublets and one singlet involved in the symmetry breaking, and one of the doublets $\left(h_{c}\right)$ does not have trilinear couplings to the singlet, so that it enters the potential only through $D$ terms. We find that while the pattern of the masses obtained gener- ally follows the pattern obtained in [14], there are additional features in the mass spectrum of the charginos, neutralinos, and Higgs scalars.

\section{Fermion masses}

With the identification of $Q_{c}$ as the quark doublet of the third family, $m_{t}=156 \mathrm{GeV}$, and $m_{b}=83 \mathrm{GeV}$, where $m_{b}$ is evaluated at $M_{Z}$. The low value for $m_{t}$, despite the high value of $v=348 \mathrm{GeV}$, is because of the low value of $\sqrt{2}\left\langle\bar{h}_{c}^{0}\right\rangle / v \sim 0.66$. Clearly, $\left\langle h_{c}^{0}\right\rangle$ is much too large for this example $^{3}$ as is reflected in the unacceptably large value for $m_{b}$. That is, the $t-b$ unification is not acceptable in the example because it would require a large ratio of $\left\langle\bar{h}_{c}^{0}\right\rangle /\left\langle h_{c}^{0}\right\rangle$. If we identify $h_{d}, h_{a}$ as the lepton doublets of the third and second families, $m_{\tau}=32 \mathrm{GeV}$, and $m_{\mu}=27 \mathrm{GeV}$, where the difference is due to the $e_{h, e}^{c} h_{e} h_{a}$ terms in $W_{3}$. The ratio $m_{b} / m_{\tau}$ is larger than in the usual $b-\tau$ unification because of the ratio $1: 1 / \sqrt{2}$ of the Yukawa couplings at the string scale, and is probably inconsistent with experiment [21]. Of course, the high value for $m_{\mu}$ is unphysical.

There is no mechanism to generate significant $u, d, c, s$, and $e^{-}$masses for this direction.

\section{Squarks and sleptons}

The squark and slepton masses take the values $m_{\tilde{t} L}$ $=2540 \mathrm{GeV}, \quad m_{\tilde{t} R}=2900 \mathrm{GeV} ; m_{\tilde{b} L}=2600 \mathrm{GeV}, m_{\tilde{b} R}$ $=2780 \mathrm{GeV} ; \quad m_{\tilde{\tau} L}=2760 \mathrm{GeV}, \quad m_{\tilde{\tau} R}=3650 \mathrm{GeV} ; m_{\tilde{\mu} L}$ $=2790 \mathrm{GeV}, m_{\tilde{\mu} R}=3670 \mathrm{GeV}$. The large values are needed to ensure a large $M_{Z^{\prime}}$ in this model. The numerical values actually refer to the mass eigenstates, which are mixtures of the $L$ and $R$ states. However, the $L-R$ mixing terms are small compared to the diagonal terms for this example; so the mixing effects are small. The other squark and slepton masses depend on initial values for the soft supersymmetry breaking mass parameters that approximately decouple from the symmetry breaking pattern, and are not presented.

\section{Charginos}

The positively charged gauginos and Higgsinos are $\left\{\widetilde{W}^{+}, \widetilde{\bar{h}}_{c}, \widetilde{\bar{h}}_{a}\right\}$, and the negatively charged gauginos and Higgsinos are $\left\{\widetilde{W}^{-}, \widetilde{h}_{c}, \widetilde{h}_{b}^{\prime}\right\}$. The mass matrix is given by

$$
M_{\tilde{\chi}^{ \pm}}=\left(\begin{array}{ccc}
M_{2} & \frac{1}{\sqrt{2}} g_{2} v_{1} & \frac{1}{\sqrt{2}} g_{2} v_{3} \\
\frac{1}{\sqrt{2}} g_{2} v_{2} & 0 & \Gamma_{s} \frac{s}{\sqrt{2}} \\
0 & 0 & 0
\end{array}\right) .
$$

\footnotetext{
${ }^{3}$ Smaller and more realistic values for $m_{b}$ and $m_{\tau}$ could have been obtained by further adjustment of parameters to yield a smaller $\left\langle h_{c}^{0}\right\rangle$. This would violate our strategy of presenting a typical model with a realistic $Z-Z^{\prime}$ hierarchy without further adjustment. Smaller $\left\langle h_{c}^{0}\right\rangle$ would also have resulted in still smaller masses for the lightest charged and scalar Higgs particles.
} 
There is one massless chargino, and the other two are massive, with masses $m_{\tilde{\chi}_{1}^{ \pm}}=591 \mathrm{GeV}$ and $m_{\tilde{\chi}_{2}^{ \pm}}=826 \mathrm{GeV}$.

The massless state involves $\bar{h}_{a}$ (and a linear combination of the negative states), and is due to the absence of $\bar{h}_{a}$ couplings in the superpotential. In particular, there is no noncanonical $\mu$ term $\bar{h}_{a} h_{c} \varphi$ in this flat direction; the only gaugeallowed term of this type $\left(\bar{h}_{a} h_{c} \varphi_{25}\right)$ was in fact in the original trilinear superpotential, but $\varphi_{25}$ has acquired a string-scale mass and decoupled from the low-energy theory. Therefore, there is no mechanism for $\bar{h}_{a}$ to acquire a VEV.

\section{Neutralinos}

The neutralino sector consists of $\left\{\widetilde{B}^{\prime}, \widetilde{B}, \widetilde{W}_{3}, \widetilde{\bar{h}}_{c}^{0}, \widetilde{h}_{c}^{0}, \widetilde{h}_{b}^{\prime 0}, \widetilde{\varphi}_{20}^{\prime}, \widetilde{\bar{h}}_{a}^{0}\right\}$. In this basis (neglecting ${\widetilde{\bar{h}_{a}}}_{a}^{0}$, which has no couplings), the neutralino mass matrix is given by

$$
M_{\tilde{\chi}^{0}}=\left(\begin{array}{ccccccc}
M_{1}^{\prime} & 0 & 0 & g_{1}^{\prime} Q_{2} v_{2} & g_{1}^{\prime} Q_{1} v_{1} & g_{1}^{\prime} Q_{3} v_{3} & g_{1}^{\prime} Q_{S} s \\
0 & M_{1} & 0 & \frac{1}{2} g_{Y} v_{2} & -\frac{1}{2} g_{Y} v_{1} & -\frac{1}{2} g_{Y} v_{3} & 0 \\
0 & 0 & M_{2} & -\frac{1}{2} g_{2} v_{2} & \frac{1}{2} g_{2} v_{1} & \frac{1}{2} g_{2} v_{3} & 0 \\
g_{1}^{\prime} Q_{2} v_{2} & \frac{1}{2} g_{Y} v_{2} & -\frac{1}{2} g_{2} v_{2} & 0 & 0 & -\frac{1}{\sqrt{2}} \Gamma_{s} s & -\frac{1}{\sqrt{2}} \Gamma_{s} v_{3} \\
g_{1}^{\prime} Q_{1} v_{1} & -\frac{1}{2} g_{Y} v_{1} & \frac{1}{2} g_{2} v_{1} & 0 & 0 & 0 & 0 \\
g_{1}^{\prime} Q_{3} v_{3} & -\frac{1}{2} g_{Y} v_{3} & \frac{1}{2} g_{2} v_{3} & -\frac{1}{\sqrt{2}} \Gamma_{s} s & 0 & 0 & -\frac{1}{\sqrt{2}} \Gamma_{s} v_{2} \\
g_{1}^{\prime} Q_{S} s & 0 & 0 & -\frac{1}{\sqrt{2}} \Gamma_{s} v_{3} & 0 & -\frac{1}{\sqrt{2}} \Gamma_{s} v_{2} & 0
\end{array} \mid .\right.
$$

The mass eigenvalues are $m_{\tilde{\chi}_{1}}^{0}=963 \mathrm{GeV}, \quad m_{\tilde{\chi}_{2}}^{0}$ $=825 \mathrm{GeV}, \quad m_{\tilde{\chi}_{3}}^{0}=801 \mathrm{GeV}, \quad m_{\tilde{\chi}_{4}}^{0}=592 \mathrm{GeV}, \quad m_{\tilde{\chi}_{5}}^{0}$ $=562 \mathrm{GeV}, m_{\tilde{\chi}_{6}}^{0}=440 \mathrm{GeV}, m_{\tilde{\chi}_{7}}^{0}=2 \mathrm{GeV}$, and $m_{\tilde{\chi}_{8}}^{0}=0$.

$\tilde{\chi}_{8}^{0}$ corresponds to $\tilde{\bar{h}}_{a}^{0}$, which (as previously mentioned) does not enter the superpotential. The hierarchy of the nonzero masses can be understood in the large singlet VEV scenario $\Gamma_{s}^{2}\left\langle\varphi_{20}^{\prime}\right\rangle^{2} \gg M_{i}^{2}, M_{Z}^{2}$ (in which $M_{i}$ denotes the gaugino masses), which yields the pattern $\tilde{\chi}_{1,2}=\left(\tilde{\bar{h}}_{c}^{0} \pm \tilde{h}_{b}^{\prime 0}\right) / \sqrt{2}$, with masses $\sim \Gamma_{s}\left\langle\varphi_{20}^{\prime}\right\rangle ; \tilde{\chi}_{3,4}=\left(\widetilde{B}^{\prime} \pm \widetilde{\varphi}_{20}^{\prime}\right) / \sqrt{2}$, with masses $\sim M_{Z}^{\prime}$; $\tilde{\chi}_{5,6}=\widetilde{B}^{\prime}, \widetilde{W}^{0}$, with masses $\sim\left|M_{1}\right|,\left|M_{2}\right|$; and $\tilde{\chi}_{7}=\widetilde{h}_{c}^{0}$, with mass $\sim 0$.

\section{Exotics}

In addition to the massless quarks, leptons, chargino, and neutralino discussed above, there are a number of exotic states, including the $S U(2)_{L}$ singlet down-type quark, four $S U(2)_{L}$ singlets with unit charge (the $e$ and extra $e^{c}$ states), and a number of SM singlet $(\varphi)$ states. There are additional exotics associated with the hidden sector. The scalar components of these exotics are expected to acquire $\mathrm{TeV}$-scale masses by soft supersymmetry breaking. However, there is no mechanism for this direction to give the fermions a sig- nificant mass. In particular, fermion masses associated with higher-dimensional operators would be suppressed by powers of the ratio of the $\mathrm{TeV}$ scale to the string scale, and are therefore negligible. (Such operators could be a viable mechanism for other flat directions that allow an intermediate scale, however.) Another possible mechanism would be to invoke a non-minimal Kähler potential. However, that is beyond the scope of the present analysis.

\section{Higgs sector}

The non-minimal Higgs sector of three complex doublets and one complex singlet required for this scenario leads to additional Higgs bosons compared to the MSSM. In this scenario, 4 of the 14 degrees of freedom are absorbed to become the longitudinal components of the $W^{ \pm}, Z$, and $Z^{\prime}$; in addition, there is a global $U(1)$ symmetry present in Eq. (11) associated with the phase of $h_{c}$ which is broken, leading to a massless Goldstone boson in the spectrum. It would acquire a small mass at the loop level due to couplings in the full theory which do not respect the global $U(1)$.

The spectrum of the physical Higgs bosons after symmetry breaking consists of two pairs of charged Higgs bosons $H_{1,2}^{ \pm}$, four neutral $C P$ even Higgs scalars $\left(h_{i}^{0}, i=1,2,3,4\right)$, and one $C P$ odd Higgs $A^{0}$. 
In the basis $\left\{\bar{h}_{c}^{0 i} \equiv \sqrt{2} \operatorname{Im} \bar{h}_{c}^{0}, h_{c}^{0 i}, h_{b}^{\prime 0 i}, s^{i}\right\}$, the $C P$ odd (tree-level) mass matrix is given by

$$
M_{A^{0}}^{2}=\frac{A \Gamma_{s}}{\sqrt{2}}\left(\begin{array}{cccc}
\frac{s v_{3}}{v_{2}} & 0 & s & v_{3} \\
0 & 0 & 0 & 0 \\
s & 0 & \frac{s v_{2}}{v_{3}} & v_{2} \\
v_{3} & 0 & v_{2} & \frac{v_{2} v_{3}}{s}
\end{array}\right) .
$$

There are one massive and three massless eigenstates. Two of the massless eigenstates are the Goldstone bosons which are absorbed to become the longitudinal components of the $Z$ and the $Z^{\prime}$. The third massless state is the Goldstone boson corresponding to the breakdown of the global $U(1)$ symmetry present in Eq. (11), due to the absence of trilinear couplings involving $h_{c}$. The physical $C P$ odd Higgs boson has

$$
m_{A^{0}}^{2}=\frac{A \Gamma_{s}}{\sqrt{2}}\left(\frac{s v_{2}}{v_{3}}+\frac{s v_{3}}{v_{2}}+\frac{v_{2} v_{3}}{s}\right)
$$

which takes the value $m_{A^{0}}=1650 \mathrm{GeV}$ in this particular case.

The mass matrix of the charged Higgs bosons in the basis $\left\{h_{c}^{-}, \bar{h}_{c}^{+*}, h_{b}^{\prime-}\right\}$ takes the form

$$
M_{H^{ \pm}}^{2}=\left(\begin{array}{ccc}
\frac{g_{2}^{2}}{4}\left(v_{2}^{2}-v_{3}^{2}\right) & \frac{g_{2}^{2}}{4} v_{1} v_{2} & \frac{g_{2}^{2}}{4} v_{1} v_{3} \\
\frac{g_{2}^{2}}{4} v_{1} v_{2} & A \Gamma_{s} \frac{s v_{3}}{\sqrt{2} v_{2}}+\frac{g_{2}^{2}}{4}\left(v_{1}^{2}+v_{3}^{2}\right)-\Gamma_{s}^{2} \frac{v_{3}^{2}}{2} & \frac{g_{2}^{2}}{4} v_{2} v_{3}-\Gamma_{s}^{2} \frac{v_{2} v_{3}}{2}+A \Gamma_{s} \frac{s}{\sqrt{2}} \\
\frac{g_{2}^{2}}{4} v_{1} v_{3} & \frac{g_{2}^{2}}{4} v_{3} v_{2}-\Gamma_{s}^{2} \frac{v_{2} v_{3}}{2}+A \Gamma_{s} \frac{s}{\sqrt{2}} & A \Gamma_{s} \frac{s v_{2}}{\sqrt{2} v_{3}}+\frac{g_{2}^{2}}{4}\left(v_{2}^{2}-v_{1}^{2}\right)-\Gamma_{s}^{2} \frac{v_{2}^{2}}{2}
\end{array}\right) .
$$

There is one massless state, which is the Goldstone boson absorbed by $W^{ \pm}$after the $S U(2)$ symmetry is spontaneously broken; the two physical charged Higgs bosons are $m_{H_{1}^{ \pm}}=10 \mathrm{GeV}$ and $m_{H_{2}^{ \pm}}=1650 \mathrm{GeV}$. The extra light mass of $H_{1}^{ \pm}$is due to an accidental cancellation between $v_{2}$ and $v_{3}$ for the specific example considered; in general, it is $\sim \mathcal{O}\left(M_{Z}\right)$.

The masses for the four neutral scalars can be obtained by diagonalizing the mass matrix (in the basis $\left\{\bar{h}_{c}^{0 r}, h_{c}^{0 r}, h_{b}^{\prime 0 r}, s^{r}\right\}$ )

$$
M_{h^{0}}^{2}=\left(\begin{array}{cccc}
\kappa_{2}^{2} v_{2}^{2}+A \Gamma_{s} s \frac{v_{3}}{\sqrt{2} v_{2}} & \kappa_{12} v_{1} v_{2} & \kappa_{23} v_{2} v_{3}-A \Gamma_{s} \frac{s}{\sqrt{2}} & \kappa_{2 s} v_{2} s-A \Gamma_{s} \frac{v_{3}}{\sqrt{2}} \\
\kappa_{12} v_{1} v_{2} & \kappa_{1}^{2} v_{1}^{2} & \kappa_{13} v_{1} v_{3} & \kappa_{1 s} v_{1} s \\
\kappa_{23} v_{2} v_{3}-A \Gamma_{s} \frac{s}{\sqrt{2}} & \kappa_{13} v_{1} v_{3} & \kappa_{3}^{2} v_{3}^{2}+A \Gamma_{s} s \frac{v_{2}}{\sqrt{2} v_{3}} & \kappa_{3 s} v_{3} s-A \Gamma_{s} \frac{v_{2}}{\sqrt{2}} \\
\kappa_{2 s} v_{2} s-A \Gamma_{s} \frac{v_{3}}{\sqrt{2}} & \kappa_{1 s} v_{1} s & \kappa_{3 s} v_{3} s-A \Gamma_{s} \frac{v_{2}}{\sqrt{2}} & \kappa_{s}^{2} s^{2}+A \Gamma_{s} v_{3} \frac{v_{2}}{\sqrt{2} s}
\end{array}\right),
$$

with $\kappa_{i}^{2}=G^{2} / 4+g^{\prime 2}{ }_{1}^{2} Q_{i}^{2}, \quad \kappa_{1 j=2,3}=g^{\prime 2}{ }_{1}^{2} Q_{1} Q_{j}-G^{2} / 4, \quad \kappa_{1 s}$ $=g_{1}^{\prime 2} Q_{1} Q_{S}, \quad \kappa_{23}=\Gamma_{s}^{2}+g^{\prime 2} Q_{1} Q_{j}-G^{2} / 4, \quad \kappa_{j s}=\Gamma_{s}^{2}$ $+g^{\prime 2} Q_{j} Q_{S}$, and $\kappa_{s}^{2}=g^{\prime 2}{ }_{1}^{2} Q_{S}^{2}$.

In the numerical solution obtained, the values of the masses of the four scalars are $m_{h_{1}^{0}}=33 \mathrm{GeV}, m_{h_{2}^{0}}$ $=47 \mathrm{GeV}, m_{h_{3}^{0}}=736 \mathrm{GeV}$, and $m_{h_{4}^{0}}=1650 \mathrm{GeV}$.

In general supersymmetric models, one of the physical Higgs bosons has a mass controlled by the electroweak scale from the breaking of $S U(2)_{L} \times U(1)_{Y}$, while the others may have masses at the scale of the soft supersymmetry (SUSY) breaking. In this model, an additional global $U(1)$ symmetry is broken when $h_{c}$ acquires a non-zero VEV, and the mass of the Higgs scalar associated with this direction is mainly de- termined by the $U(1)$ breaking scale. This scale is therefore comparable to the electroweak scale, which indicates that in the decoupling limit not only one but two Higgs scalars will be light. In this particular example, the VEV of $h_{c}$ happens to be small compared with that of the other two Higgs doublets, and hence the lightest Higgs boson is mainly associated with $h_{c}$. Thus, the lightest Higgs boson mass satisfies the (tree-level) bound

$$
m_{h_{1}^{0}}^{2} \leqslant \frac{G^{2}}{4} v_{1}^{2}+g_{1}^{2}, Q_{1}^{2} v_{1}^{2}=(35 \mathrm{GeV})^{2},
$$

obtained from analyzing the potential in the field direction 
that breaks the global $U(1)$ symmetry (i.e., the field direction $h_{c}$ ) [22]. In the large $s$ limit, the mass saturates this bound.

It is also possible to place a bound on the second-lightest neutral Higgs scalar [22]

$$
\begin{aligned}
m_{h_{2}^{0}}^{2} \leqslant & m_{h_{1}^{0}}^{2}+\frac{v^{2}}{v_{2}^{2}+v_{3}^{2}}\left[\left(\frac{G^{2} v_{1}^{2}}{4}+g_{1^{\prime}}^{2} Q_{1}^{2} v_{1}^{2}-m_{h_{1}^{0}}^{2}\right)^{1 / 2}\right. \\
& +\left(\frac{G^{2}}{4 v^{2}}\left(v_{2}^{2}-v_{1}^{2}-v_{3}^{2}\right)^{2}+\frac{g_{1^{\prime}}^{2}}{v^{2}}\left(Q_{1} v_{1}^{2}+Q_{2} v_{2}^{2}+Q_{3} v_{3}^{2}\right)^{2}\right. \\
& \left.\left.+2 \frac{\Gamma_{s}^{2}}{v^{2}} v_{2}^{2} v_{3}^{2}-m_{h_{1}^{0}}^{2}\right)^{1 / 2}\right]^{2}=(85 \mathrm{GeV})^{2}
\end{aligned}
$$

A suitable rotation in field space demonstrates that the second-lightest Higgs boson $\left(h_{2}^{0}\right)$ is basically the real part of the Higgs doublet that is involved in the $S U(2)$ breaking, while the other two Higgs doublets do not participate. One of the two rotated doublets $\left(h_{4}^{0}, H_{2}^{ \pm}, A^{0}\right)$ consists of the heaviest scalar, pseudoscalar, and charged Higgs boson, and is composed mainly of $\bar{h}_{c}$ and $h_{b}^{\prime}$, with mass roughly given by $m_{A}^{0}$ (naturally expected to be large in this limit). The other doublet $\left(h_{1}^{0}, H_{1}^{ \pm}\right.$, massless pseudoscalar) is basically $h_{c}$, and hence the associated fields are light due to the absence of couplings to the singlet. The second-heaviest neutral Higgs boson $\left(h_{3}^{0}\right)$ has mass governed by $M_{Z^{\prime}}$, and is primarily the singlet.

\section{IV. $\left.P_{2} P_{3}\right|_{F}$ FLAT DIRECTION}

\section{A. Effective superpotential}

The fields involved in the $\left.P_{2} P_{3}\right|_{F}$ direction are $\left\{\varphi_{2}, \varphi_{4}, \varphi_{10}, \varphi_{12}, \varphi_{27}, \varphi_{30}\right\}$, with VEV's
TABLE V. List of massless states for the $\left.P_{2} P_{3}\right|_{F}$ flat direction, where $N_{1 / 2} \equiv g^{2} x^{2} / 4+\left(\alpha_{5}^{(1 / 2)} x^{3} / 2 M_{P l}^{2}\right)^{2}$.

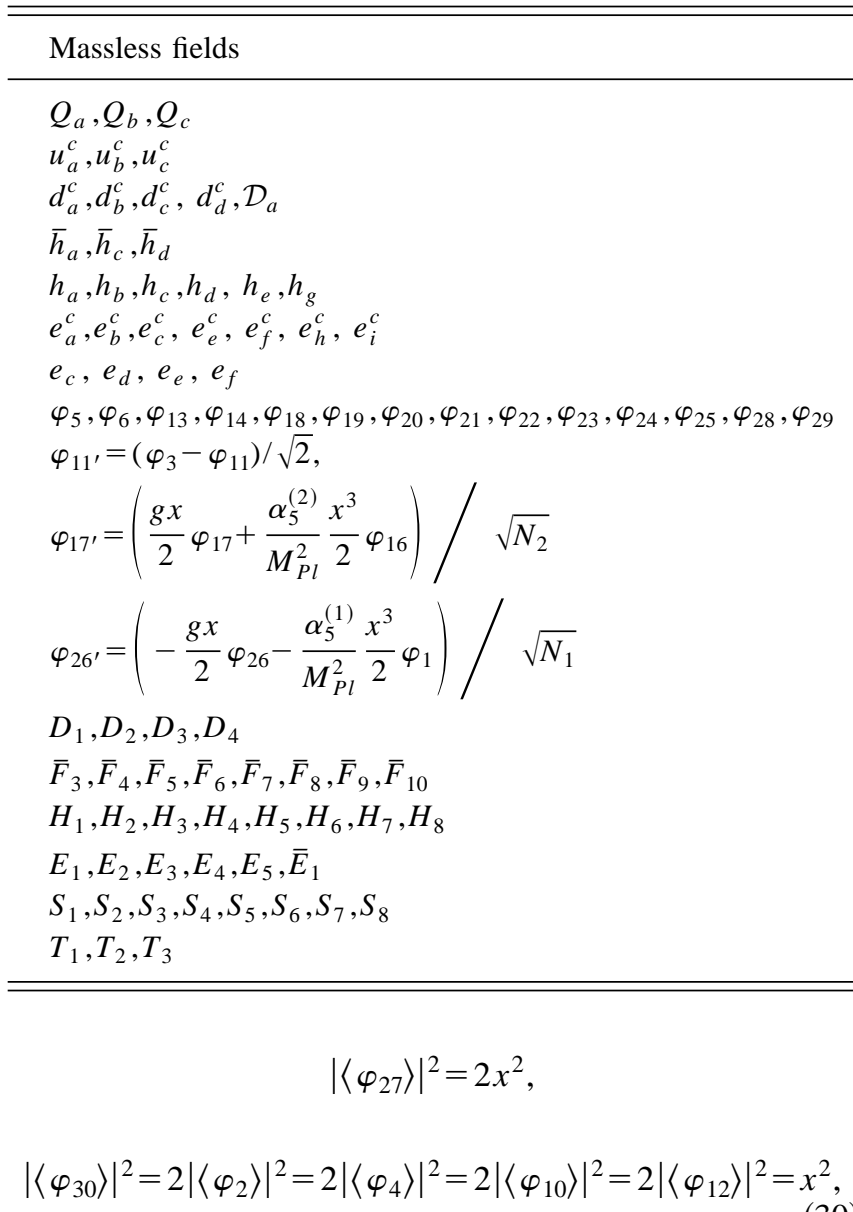

where $x=0.013 M_{P l}$, and $\left\langle\varphi_{10}\right\rangle$ and $\left\langle\varphi_{4}\right\rangle$ have opposite signs.

The massless states are presented in Table V. The effective trilinear couplings for the observable sector states are given by

$$
\begin{aligned}
W_{3}= & g Q_{c} u_{c}^{c} \bar{h}_{c}+g Q_{c} d_{b}^{c} h_{c}+\frac{\alpha_{5}^{(3)} x^{2}}{\sqrt{2} M_{P l}^{2}} Q_{a} d_{d}^{c} h_{g}+\frac{\alpha_{5}^{(4)} x^{2}}{\sqrt{2} M_{P l}^{2}} Q_{b} d_{d}^{c} h_{g}+\frac{\alpha_{5}^{(5)} x^{2}}{\sqrt{2} M_{P l}^{2}} Q_{b} d_{c}^{c} h_{b}+\frac{\alpha_{5}^{(6)} x^{2}}{\sqrt{2} M_{P l}^{2}} Q_{a} d_{c}^{c} h_{b} \\
& +\frac{\sqrt{\left(\alpha_{4}^{(1)}\right)^{2}+\left(\alpha_{4}^{\left(1^{\prime}\right)}\right)^{2} x}}{\sqrt{2} M_{P l}} u_{b}^{c^{\prime}} d_{c}^{c} d_{d}^{c}+\frac{g}{\sqrt{2}} e_{a}^{c} h_{a} h_{c}+\frac{g}{\sqrt{2}} e_{f}^{c} h_{d} h_{c}+\frac{\sqrt{\left(\alpha_{4}^{(2)}\right)^{2}+\left(\alpha_{4}^{\left(2^{\prime}\right)}\right)^{2} x}}{\sqrt{2} M_{P l}} e_{e}^{c^{\prime}} h_{g} h_{b}+\frac{\alpha_{5}^{(7)} x^{2}}{2 M_{P l}^{2}} e_{b}^{c} h_{g} h_{a} \\
& +\frac{\alpha_{5}^{(8)} x^{2}}{2 M_{P l}^{2}} e_{i}^{c} h_{g} h_{a}+\frac{g}{\sqrt{2}} \bar{h}_{a} h_{c} \varphi_{25}+g \bar{h}_{c} h_{b} \varphi_{20}+g \bar{h}_{d} h_{b} \varphi_{28}+g \bar{h}_{c} h_{g} \varphi_{21}+g \bar{h}_{d} h_{g} \varphi_{29}+\frac{\alpha_{4}^{(3)} \sqrt{2} x}{M_{P l}} \varphi_{25} \varphi_{21} \varphi_{29} \\
& +\frac{\alpha_{4}^{\left(3^{\prime}\right)} \sqrt{2} x}{M_{P l}} \varphi_{25} \varphi_{20} \varphi_{28},
\end{aligned}
$$


in which

$$
\begin{aligned}
& u_{b}^{c^{\prime}}=\frac{M_{P l}}{\sqrt{\left(\alpha_{4}^{(1)}\left\langle\varphi_{4}\right\rangle\right)^{2}+\left(\alpha_{4}^{\left(1^{\prime}\right)}\left\langle\varphi_{12}\right\rangle\right)^{2}}} \\
& \times\left(\frac{\alpha_{4}^{(1)}}{M_{P l}}\left\langle\varphi_{4}\right\rangle u_{b}^{c}+\frac{\alpha_{4}^{\left(1^{\prime}\right)}}{M_{P l}}\left\langle\varphi_{12}\right\rangle u_{a}^{c}\right), \\
& e_{e}^{c^{\prime}}=\frac{M_{P l}}{\sqrt{\left(\alpha_{4}^{(2)}\left\langle\varphi_{12}\right\rangle\right)^{2}+\left(\alpha_{4}^{\left(2^{\prime}\right)}\left\langle\varphi_{4}\right\rangle\right)^{2}}} \\
& \times\left(\frac{\alpha_{4}^{(2)}}{M_{P l}}\left\langle\varphi_{12}\right\rangle e_{e}^{c}+\frac{\alpha_{4}^{\left(2^{\prime}\right)}}{M_{P l}}\left\langle\varphi_{4}\right\rangle e_{h}^{c}\right) .
\end{aligned}
$$

The superpotential (31) displays the same unrealistic $t-b$ and $\tau$ - $\mu$ unification, absence of the canonical effective $\mu$ term (though non-canonical $\mu$ terms are present), and $L$-violating couplings as in the previous case.

However, there are new features (see [3] for a detailed discussion). In particular, there are $B$-number violating couplings in the superpotential, with implications for possible proton decay processes and $N-\bar{N}$ oscillations. There is also a texture in the down-quark sector, with a possibly realistic $m_{s} / m_{b}$ ratio due to the contribution of the original fifth order operators.

An inspection of Table $\mathrm{V}$ shows that there are, in fact, a larger number of massless states in the observable sector than in the $P_{1}^{\prime} P_{2}^{\prime} P_{3}^{\prime}$ flat direction. Once again, there is some ambiguity in how to identify the three MSSM lepton doublets (each possible set from the list of massless states leads to $L$-violating couplings). However, in this case there is an additional pair of fields $\left(\bar{h}_{d}, h_{g}\right)$ which can play the role of Higgs doublets.

In addition, $\varphi_{25}$ remains massless at the string scale in this model. Therefore, there is a possibility that the $U(1)^{\prime}$ breaking may occur along a $D$ flat direction, and hence takes place at an intermediate scale. This scenario requires that the mass square of the field relevant along the flat direction be driven negative at a scale much higher than the electroweak scale. We investigate this possibility in Sec. IV C, and show that it is possible with mild tuning of the soft supersymmetry breaking parameters at the string scale. On the other hand, if this condition is not satisfied, the $U(1)^{\prime}$ symmetry breaking is naturally at the electroweak scale, coupled to the breaking of $S U(2)_{L} \times U(1)_{Y}$; we examine this possibility in the following subsection.

As in the previous model, we adopt the strategy that the initial boundary conditions for the hidden sector fields are adjusted to keep their mass squares positive at the observable sector symmetry breaking scale. (In this case, the hidden sector is more involved since the singlet field $\varphi_{21}$ couples both to the hidden sector fields and the Higgs doublets [Eq. (27) in [3]].)

\section{B. Electroweak scale symmetry breaking}

To highlight the unique features of the $\left.P_{2} P_{3}\right|_{F}$ case as compared with the previous example, we choose to study scenarios which allow for the maximum amount of texture in the quark sector, i.e., scenarios in which the Higgs doublets $\bar{h}_{c}, h_{c}, h_{g}$ and $h_{b}$, as well as the singlet fields $\varphi_{20}$ and $\varphi_{21}$, all acquire non-zero VEV's. ${ }^{4}$ We again restrict ourselves to scenarios which lead to experimentally allowed $Z^{\prime}$ masses and $Z-Z^{\prime}$ mixing angles.

With these assumptions, an inspection of the resulting scalar potential reveals that not all of the phases of the fields which acquire VEV's can be eliminated by suitable rotations. By redefining the phases of the fields, the Yukawa coupling and the $A$ parameter associated with the coupling $\bar{h}_{c} h_{b} \varphi_{20}$ can be taken to be real and positive. In the most general case, $\left\langle\bar{h}_{c}^{0}\right\rangle$ and $\left\langle\varphi_{20}\right\rangle$ can be chosen to be real and positive by $S U(2)_{L} \times U(1)_{Y}$ and $U(1)^{\prime}$ rotations, respectively. Similarly, $\left\langle h_{c}^{0}\right\rangle$ and $\left\langle\varphi_{21}\right\rangle$ can be taken to be real and positive using global $U(1)$ symmetries of the scalar potential. Therefore, there are in general three phases that will remain nonzero at the minimum of the potential: $\phi_{b}, \phi_{g}$ and $\phi_{A_{2}}$, which are the phases of $\left\langle h_{b}\right\rangle,\left\langle h_{g}\right\rangle$, and the $A$ parameter associated with the coupling $\bar{h}_{c} h_{g} \varphi_{21}$. We will take the $A$ parameter to be real and positive (i.e., we ignore possible explicit $C P$ violation associated with the soft supersymmetry breaking). $\phi_{b}$ and $\phi_{g}$ may be non-zero at the minimum of the potential, leading to spontaneous $C P$ violation and the associated difficulties of cosmological domain walls. However, $\phi_{b}$ and $\phi_{g}$ vanish at the minimum for the particular numerical example that we consider.

\section{Running of the gauge couplings and Yukawa couplings}

We adopt the same strategy for the running of the gauge couplings as the previous model. The $\beta$ functions for the gauge groups are presented in Table III. At the electroweak scale, one obtains $g_{1}=0.40$ (which includes the factor $k_{Y}$ $=11 / 3)$ and $g_{2}=0.46\left(k_{2}=1\right)$, yielding $\sin ^{2} \theta_{W}=0.17$.

This model displays a rich set of Yukawa couplings for the observable sector. The initial values of the coupling constants are fixed by string calculations. In Fig. 4, we show the variation of the coupling constants with the scale.

\footnotetext{
${ }^{4}$ It is also possible that $\bar{h}_{a}, \bar{h}_{d}$, and the singlet fields $\varphi_{25}, \varphi_{28}$, and $\varphi_{29}$ acquire non-zero VEV's, due to the presence of the noncanonical effective $\mu$ terms involving these fields, if the singlet fields involved develop negative mass squares at the electroweak scale. With the non-canonical $\mu$ terms $\bar{h}_{a} h_{c} \varphi_{25}, \bar{h}_{d} h_{b} \varphi_{28}$ and $\bar{h}_{d} h_{g} \varphi_{29}$ taking active roles in the symmetry breaking, the massless charginos and neutralinos (associated with the Higgs doublets that do not have VEV's) as well as the massless $C P$ odd Higgs scalars (associated with the Higgs doublets that do not have effective $\mu$ terms) can be eliminated. However, this implies that the minimum of the potential has a very complicated structure, which may result in a larger amount of fine-tuning. We do not investigate such complicated scenarios in this paper.
} 


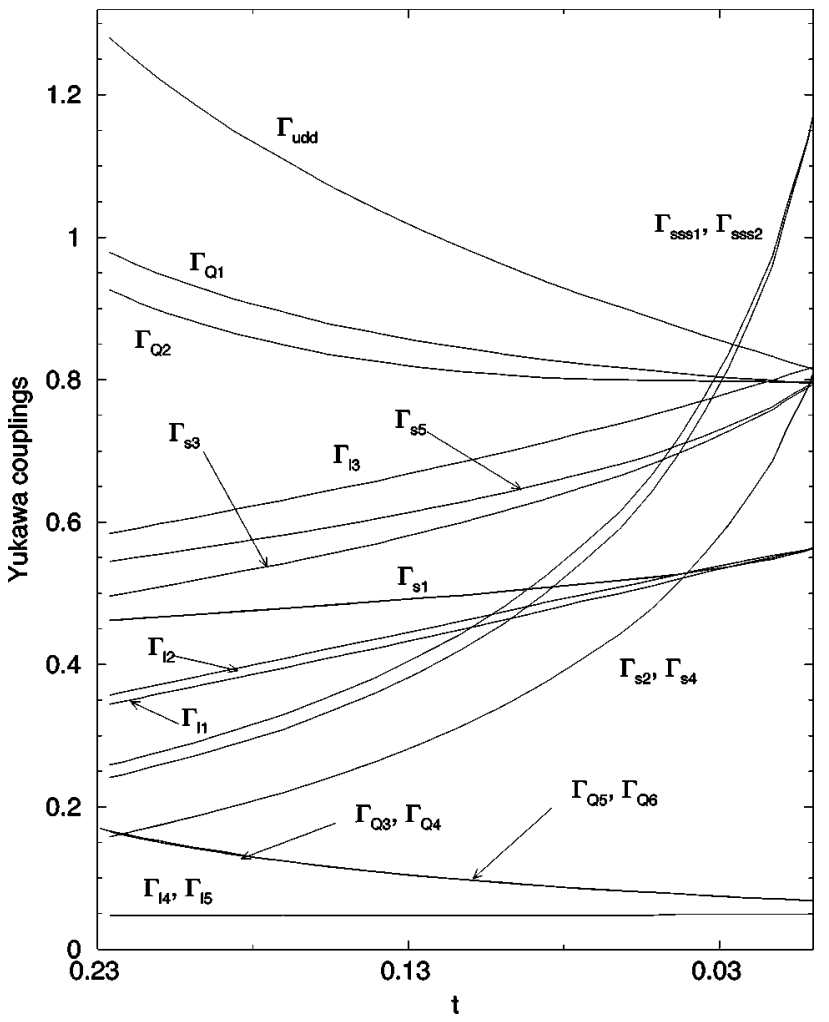

FIG. 4. Running of the Yukawa couplings for the $\left.P_{2} P_{3}\right|_{F}$ direction. The naming and the ordering of the Yukawa coupling constants are the same as in the previous model. $\Gamma_{u d d}$ refers to the $u_{b}^{c^{\prime}} d_{c}^{c} d_{d}^{c}$ coupling; $\Gamma_{s s s 1,2}$ refer to the last two terms in Eq. (31).

In addition to the large Yukawa couplings of $\bar{h}_{c}$ and $h_{c}$ to the top and bottom quarks (which take the values $\Gamma_{Q 1}$ $=0.98$ and $\Gamma_{Q 2}=0.93$ at the electroweak scale), the effective Yukawa couplings from the fifth order (which involve the other two quark families) have the non-trivial values $\Gamma_{Q 3}$ $=\Gamma_{Q 4}=\Gamma_{Q 5}=\Gamma_{Q 6}=0.17$. With the assumption that $h_{g}$ and $h_{b}$ have non-zero VEV's, these couplings naturally provide a hierarchy of the quark masses and mixings in the down-type quark sector.

The $\tau-\mu$ unification is slightly broken, since $\Gamma_{l 1}=0.34$ and $\Gamma_{l 2}=0.35$ at the electroweak scale. The Yukawa couplings of the non-canonical $\mu$ terms are non-trivial as well, with typical values of a few times 0.1 at low energy. Hence, these couplings can be actively involved in the symmetry breaking, as will be manifest in our numerical example.

\section{Running of the soft mass parameters}

The mass squares of $h_{g}$ and $h_{b}$ tend to remain positive, as their couplings to quarks arise from fifth order terms in the original superpotential and are therefore suppressed. However, the coupling $\bar{h}_{c} h_{g} \varphi_{21}$ may force $h_{g}$ to acquire a nonzero VEV. Similarly, $h_{b}$ may acquire a VEV due to the $\bar{h}_{c} h_{b} \varphi_{20}$ coupling. There are then two distinct electroweak symmetry breaking patterns, one in which $h_{b}$ has a non-zero VEV (i.e., $h_{b}$ is naturally identified as a Higgs field) and one in which $h_{b}$ has zero VEV ( $h_{b}$ could also be identified as a lepton doublet).
With universal soft mass-squared parameters at the string scale, the soft mass-squared parameters of $\bar{h}_{c}$ and $h_{c}$ are driven to negative values, while the mass squares of all the other fields remain positive. This scenario results in a light $Z^{\prime}$ and large $Z-Z^{\prime}$ mixing angle at the electroweak scale, which is excluded by experiments. We therefore have to consider non-universal boundary conditions for a realistic solution, in which $h_{g}, h_{b}, \varphi_{20}$, and $\varphi_{21}$ also acquire VEV's. As in the previous example, the effects of the Yukawa coupling involving hidden sector fields [Eq. (27) of [3]] are included in the running Yukawas and soft parameters.

One numerical example we found involves a set of tuned initial conditions which do not deviate substantially from universality. In Table VI, we present the numerical values of the parameters for this example, in which the VEV's of the fields $\quad$ are $\left\langle\bar{h}_{c}^{0}\right\rangle=223 \mathrm{GeV}, \quad\left\langle h_{c}^{0}\right\rangle=124 \mathrm{GeV}, \quad\left\langle h_{b}^{0}\right\rangle$ $=17.3 \mathrm{GeV},\left\langle h_{g}^{0}\right\rangle=24.8 \mathrm{GeV},\left\langle\varphi_{20}\right\rangle=24.8 \mathrm{GeV}$, and $\left\langle\varphi_{21}\right\rangle=4950 \mathrm{GeV}$. In this particular example with $A_{2}$ real and positive, $\phi_{b}=\phi_{g}=0$ at the true minimum of the potential, and hence there is no spontaneous $C P$ violation. For these values, $M_{Z^{\prime}}=1.00 \mathrm{TeV}$ and $\alpha_{Z-Z^{\prime}}=0.004$.

We now consider the quark masses and mixings in this model. The mass matrix for the down-type quarks is [3]

$$
\begin{aligned}
M & =\left(\begin{array}{ccc}
\Gamma_{Q 3}\left\langle h_{g}^{0}\right\rangle & \Gamma_{Q 6}\left\langle h_{b}^{0}\right\rangle & 0 \\
\Gamma_{Q 4}\left\langle h_{g}^{0}\right\rangle & \Gamma_{Q 5}\left\langle h_{b}^{0}\right\rangle & 0 \\
0 & 0 & \Gamma_{Q 2}\left\langle h_{c}^{0}\right\rangle
\end{array}\right) \\
& =\left(\begin{array}{ccc}
4.16 & 3.04 & 0 \\
4.16 & 3.04 & 0 \\
0 & 0 & 115
\end{array}\right),
\end{aligned}
$$

after the electroweak scale symmetry breaking. The masses of the down-type quarks are $m_{d}=0 \mathrm{GeV}, m_{s}=7 \mathrm{GeV}$, and $m_{b}=115 \mathrm{GeV}$, where $d, s$ and $b$ stand for the down, strange, and bottom quarks, respectively. These are running masses evaluated at $M_{Z}$. Just as in the example in Sec. III for the $P_{1}^{\prime} P_{2}^{\prime} P_{3}^{\prime}$ direction, the scale for $m_{s}$ and $m_{b}$ (as well as for $m_{\tau}$ and $m_{\mu}$ ) is much too high. Again, we have made no attempt to further adjust the parameters to obtain a lower $\left\langle h_{c}^{0}\right\rangle$. However, the hierarchy of the relative masses, $\left(m_{d}: m_{s}: m_{b}\right)$ $\sim(0: 1: 17)$ is quite encouraging. This hierarchy, as well as the form of the Cabibbo-Kobayashi-Maskawa (CKM) matrix, can be understood from the analytic discussion in [3]. The CKM matrix, obtained from diagonalizing $M M^{\dagger}$, since there is no contribution from the up-quark sector, is given by

$$
U^{\mathrm{CKM}}=\left(\begin{array}{ccc}
0.71 & 0.71 & 0 \\
-0.71 & 0.71 & 0 \\
0 & 0 & 1.00
\end{array}\right) .
$$

The maximal mixing between the first two families is not realistic. It results from the comparable magnitude of the $\alpha_{5}^{(i)}$ coefficients. As discussed in [3], one can obtain a realistic $m_{d} / m_{s} \neq 0$ and a realistic Cabibbo-like mixing by allowing $\alpha_{5}^{(3)} \neq \alpha_{5}^{(4)}$ and $\quad \alpha_{5}^{(5)} \neq \alpha_{5}^{(6)}$. The observed relation $\theta_{c}$ 
TABLE VI. $\left.P_{2} P_{3}\right|_{F}$ flat direction: values of the parameters (for the observable sector) at $M_{\text {String }}$ and $M_{Z}$, with $M_{Z^{\prime}}=1008 \mathrm{GeV}$ and $\alpha_{Z-Z^{\prime}}=0.004$. All mass parameters are given in $\mathrm{GeV}$.

\begin{tabular}{|c|c|c|c|c|c|}
\hline & $M_{Z}$ & $M_{\text {String }}$ & & $M_{Z}$ & $M_{\text {String }}$ \\
\hline$g_{1}$ & 0.40 & 0.80 & $M_{1}$ & 251 & 1000 \\
\hline$g_{2}$ & 0.46 & 0.80 & $M_{2}$ & 330 & 1000 \\
\hline$g_{3}$ & 1.23 & 0.80 & $M_{3}$ & 2380 & 1000 \\
\hline$g_{1}^{\prime}$ & 0.42 & 0.80 & $M_{1}^{\prime}$ & 225 & 1000 \\
\hline$\Gamma_{Q 1}$ & 0.98 & 0.80 & $A_{Q 1}$ & 828 & 702 \\
\hline$\Gamma_{Q 2}$ & 0.93 & 0.80 & $A_{Q 2}$ & 2120 & 1227 \\
\hline$\Gamma_{Q 3}$ & 0.17 & 0.07 & $A_{Q 2}$ & 3290 & 1510 \\
\hline$\Gamma_{Q 4}$ & 0.17 & 0.07 & $A_{Q 2}$ & 3290 & 1510 \\
\hline$\Gamma_{Q 5}$ & 0.17 & 0.07 & $A_{Q 2}$ & 4570 & 1308 \\
\hline$\Gamma_{Q 6}$ & 0.17 & 0.07 & $A_{Q 2}$ & 4570 & 1308 \\
\hline$\Gamma_{u d d}$ & 1.29 & 0.82 & $A_{u d d}$ & 2910 & 3239 \\
\hline$\Gamma_{l 1}$ & 0.34 & 0.56 & $A_{l 1}$ & -240 & 1234 \\
\hline$\Gamma_{l 2}$ & 0.35 & 0.56 & $A_{l 2}$ & -197 & 1234 \\
\hline$\Gamma_{l 3}$ & 0.58 & 0.82 & $A_{l 3}$ & 419 & 1659 \\
\hline$\Gamma_{l 4}$ & 0.05 & 0.05 & $A_{l 4}$ & 807 & 1351 \\
\hline$\Gamma_{l 5}$ & 0.05 & 0.05 & $A_{l 4}$ & 807 & 1351 \\
\hline$\Gamma_{s 1}$ & 0.46 & 0.56 & $A_{s 1}$ & 893 & 1381 \\
\hline$\Gamma_{s 2}$ & 0.16 & 0.80 & $A_{1}$ & 350 & 3336 \\
\hline$\Gamma_{s 3}$ & 0.49 & 0.80 & $A_{s 3}$ & 394 & 1357 \\
\hline$\Gamma_{s 4}$ & 0.16 & 0.80 & $A_{2}$ & 3500 & 6789 \\
\hline$\Gamma_{s 5}$ & 0.54 & 0.80 & $A_{s 5}$ & 361 & 1351 \\
\hline$\Gamma_{s s s 1}$ & 0.24 & 1.17 & $A_{s s s 1}$ & -938 & 1477 \\
\hline$\Gamma_{s s s 2}$ & 0.26 & 1.17 & $A_{s s s 2}$ & -763 & 1671 \\
\hline$m_{Q}^{2}$ & $(1890)^{2}$ & $(2252)^{2}$ & $m_{Q_{b}}^{2}$ & $(3110)^{2}$ & $(1934)^{2}$ \\
\hline$m_{Q_{a}}^{2^{c}}$ & $(3110)^{2}$ & $(1934)^{2}$ & $m_{u_{c}}^{2^{b}}$ & $(2010)^{2}$ & $(2352)^{2}$ \\
\hline$m_{u_{i}^{\prime}}^{2^{a}}$ & $(1460)^{2}$ & $(4382)^{2}$ & $m_{d_{b}}^{2^{c}}$ & $(1500)^{2}$ & $(2172)^{2}$ \\
\hline$m_{d_{d}}^{2^{b}}$ & $(4370)^{2}$ & $(6155)^{2}$ & $m_{d_{c}}^{2^{b}}$ & $(1460)^{2}$ & $(4382)^{2}$ \\
\hline$m_{\bar{h}}^{2^{2}}$ & $(531)^{2}$ & $(4624)^{2}$ & $m_{\bar{h}}^{2}$ & $(1040)^{2}$ & $(4549)^{2}$ \\
\hline$m_{\bar{h}}^{2^{c}}$ & $(1250)^{2}$ & $(1817)^{2}$ & $m_{h_{a}}^{2^{d}}$ & $(1390)^{2}$ & $(1828)^{2}$ \\
\hline$m_{h_{c}}^{2^{a}}$ & $-(311)^{2}$ & $(4177)^{2}$ & $m_{h_{d}}^{2^{2}}$ & $(1400)^{2}$ & $(1833)^{2}$ \\
\hline$m_{h_{g}}^{2^{c}}$ & $(4950)^{2}$ & $(7639)^{2}$ & $m_{h_{h}}^{2^{a}}$ & $-(299)^{2}$ & $(4939)^{2}$ \\
\hline$m_{e_{a}}^{2^{g}}$ & $(1920)^{2}$ & $(2590)^{2}$ & $m_{e_{f}}^{2^{b}}$ & $(1890)^{2}$ & $(2590)^{2}$ \\
\hline$m_{e_{b}}^{2^{a}}$ & $(1750)^{2}$ & $(1764)^{2}$ & $m_{e^{\prime}}^{2^{f}}$ & $(1500)^{2}$ & $(5314)^{2}$ \\
\hline$m_{e_{j}}^{2^{b}}$ & $(1750)^{2}$ & $(1764)^{2}$ & $m_{\varphi_{20}}^{2^{e}}$ & $-(715)^{2}$ & $(5177)^{2}$ \\
\hline$m_{\varphi_{21}}^{2^{i}}$ & $-(718)^{2}$ & $(6328)^{2}$ & $m_{\varphi_{25}}^{200}$ & $(1630)^{2}$ & $(4337)^{2}$ \\
\hline$m_{\varphi_{28}^{21}}^{21}$ & $(4470)^{2}$ & $(5982)^{2}$ & $m_{\varphi_{29}}^{25}$ & $(2210)^{2}$ & $(6398)^{2}$ \\
\hline
\end{tabular}

$\sim\left(m_{d} / m_{s}\right)^{1 / 2}(\sim 0.2)$ can be obtained for specific $\alpha_{5}$ 's, but does not hold in general. $U_{31}^{\mathrm{CKM}}=U_{32}^{\mathrm{CKM}}=U_{13}^{\mathrm{CKM}}=U_{23}^{\mathrm{CKM}}=0$ due to the form of $M$.

We do not present the Higgs, chargino, or neutralino spectra for this example, as they do not exhibit any qualitatively new features compared to the $P_{1}^{\prime} P_{2}^{\prime} P_{3}^{\prime}$ direction.

\section{Intermediate scale symmetry breaking}

We now investigate the possibility that the $U(1)^{\prime}$ is broken at an intermediate scale, along a $D$ flat direction. This is possible in this model because the field $\varphi_{25}$, which has a $U(1)^{\prime}$ charge opposite in sign to the rest of the singlets [with nonzero $U(1)^{\prime}$ charges], remains massless at the string scale. The viability of this scenario requires that the $D$ flat direction be also $F$ flat at the trilinear order [15]; otherwise, the $F$ terms lead to quartic couplings in the scalar potential which force the VEV's to be at the electroweak scale. ${ }^{5}$ In addition, we require the absence of renormalizable couplings of the fields in the $D$ flat direction to the Higgs doublet $\left(\bar{h}_{c}\right)$ which couples to the top quark, so that there is top quark Yukawa coupling in the low energy theory.

With these criteria in mind, an inspection of Table $\mathrm{V}$ and Eq. (31) demonstrates that from the list of singlet fields $\left(\varphi_{18}-\varphi_{22}\right)$ that have $U(1)^{\prime}$ charges equal and opposite to $\varphi_{25}$, the trilinear couplings of $\varphi_{20}$ and $\varphi_{21}$ (to $\bar{h}_{c}$ ) do not allow for a realistic implementation of the intermediate scale symmetry breaking. However, the fields $\left\{\varphi_{18}, \varphi_{19}, \varphi_{22}\right\}$ (which do not have effective trilinear couplings) can be involved in viable intermediate scale scenarios.

To achieve the $U(1)^{\prime}$ breaking, the effective mass square $m_{\varphi_{25}}^{2}+m_{\varphi_{18 / 19 / 22}}^{2}$ must be driven negative at a scale higher than the electroweak scale. With non-universal boundary conditions, the mass square of $\varphi_{25}$ can be driven negative, while keeping the mass squares of $\varphi_{20}$ and $\varphi_{21}$ positive. The mass squares of $\varphi_{18,19,22}$ do not run, due to the absence of Yukawa couplings involving these fields. Therefore, we can choose initial values of these mass squares such that $m_{\varphi_{25}}^{2}$ $+m_{\varphi_{18 / 19 / 22}}^{2}$ is driven negative at an intermediate scale (while keeping $m_{\varphi_{25}}^{2}+m_{\varphi_{20 / 21}}^{2}$ positive). These conditions ensure that the minimum occurs along the $D$ flat direction involving $\varphi_{25}$ and $\varphi_{18 / 19 / 22}$. A range of intermediate scales $\mu_{R A D}$, at which $m_{\varphi_{25}}^{2}+m_{\varphi_{18 / 19 / 22}}^{2}$ crosses zero, can be obtained by adjusting the initial values of the parameters. In Fig. 5 we display the scale variation of the mass squares with initial values that lead to $\mu_{R A D} \sim 10^{12} \mathrm{GeV}$. This example requires nonuniversal initial values, with $m_{\varphi_{20,21}}^{2}$ larger by factors of around 2 9 than the others.

In the intermediate scale symmetry breaking scenario [15], the potential can be stabilized by radiative corrections or by nonrenormalizable self-couplings of the singlet fields in the flat direction, i.e., terms of the form $\left(\varphi_{25} \varphi_{18 / 19 / 22}\right)^{n} / M^{2 n-3}$. Such nonrenormalizable selfcouplings have a number of sources. For example, they can be present in the original superpotential or induced by vacuum restabilization from higher-dimensional operators. In addition, these terms can arise from the decoupling of the heavy fields [12], as well as from a nonminimal Kähler potential (either from the original couplings from the effective string theory or be induced either after vacuum restabilization or due to decoupling effects).

\footnotetext{
${ }^{5}$ In principle, it is possible that total singlets can acquire intermediate scale VEV's, because the absence of $D$ terms leads to a similar situation as the case that arises for a $D$ flat direction. In this model, the relevant singlets $\varphi_{28,29}$ also couple to $\varphi_{25}$, which provide effective $F$ terms that push the VEV's to the electroweak scale, and hence this scenario is not viable [3].
} 


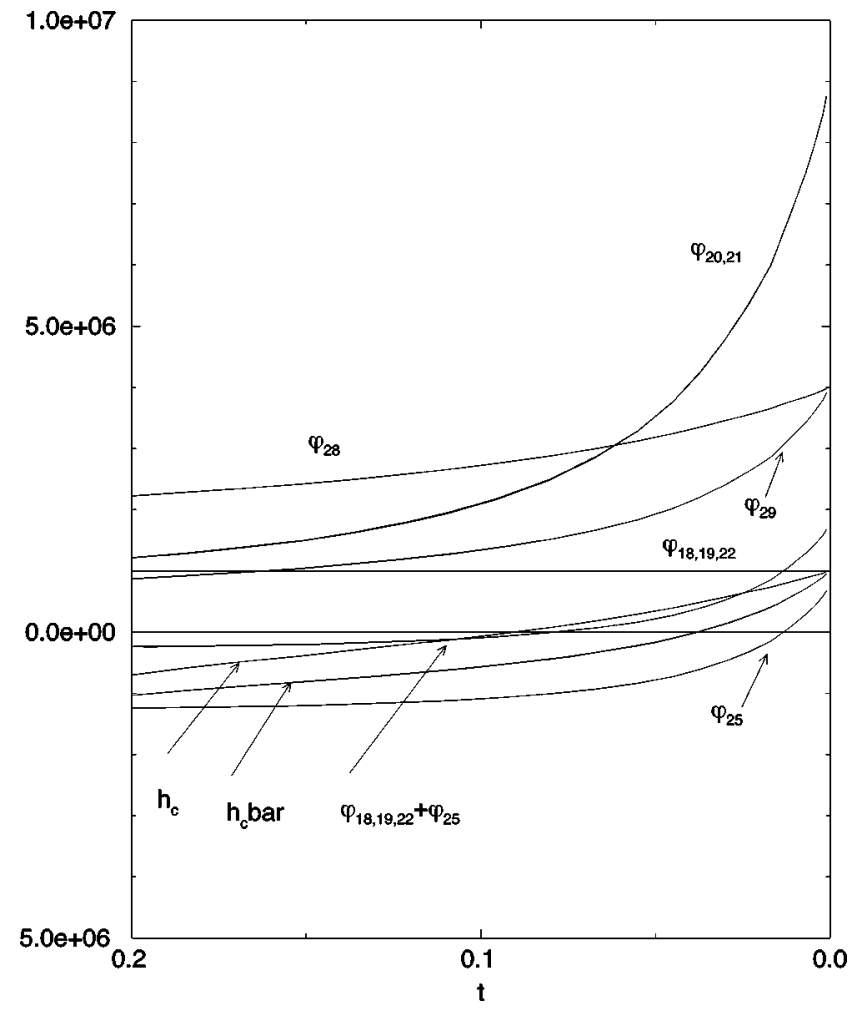

FIG. 5. Running of the relevant mass-squared parameters for the $\left.P_{2} P_{3}\right|_{F}$ flat direction, for the $U(1)^{\prime}$ symmetry breaking scenario along the $D$ flat direction $\varphi_{25}+\varphi_{18 / 19 / 22}$, with $\mu_{R A D} \sim 10^{12} \mathrm{GeV}$.

Within our assumption of a minimal Kähler potential, we have checked to determine if these nonrenormalizable selfcouplings are in fact present and found that these couplings do not survive the string selection rules [3]. To determine if such terms can be induced at the leading order in the superpotential from decoupling [12], it is necessary to check that there are no trilinear terms in the effective superpotential that involve two powers of the fields in the intermediate scale flat direction and one power of a heavy field. In this case, gauge invariance restricts these terms to be of the type $\varphi_{25} \varphi_{18 / 19 / 22} \varphi$, in which $\varphi$ is a heavy gauge singlet under $U(1)_{Y}$ and $U(1)^{\prime}$ ( $\varphi$ can be one of the fields in the $\left.P_{2} P_{3}\right|_{F}$ flat direction). We find that in this example there are no such terms. Therefore, the symmetry breaking is purely radiative in origin, and the VEV's are very close to the scale $\mu_{R A D}$.

The next step is to investigate the electroweak symmetry breaking after the intermediate scale $U(1)^{\prime}$ breaking. The electroweak symmetry breaking has different features than in the previous case. For example, the field $h_{c}$, which played an important role in the electroweak symmetry breaking due to its Yukawa couplings to the bottom quark and the $\mu$ and $\tau$ leptons, acquires an intermediate scale mass and decouples from the theory, which seems to leave these MSSM fields massless. However, it may be possible that effective mass for the exotics and effective Yukawa couplings for the MSSM fields are generated from nonrenormalizable operators involving the fields in the intermediate scale [15]. Similarly, effective $\mu$ terms may be generated by non-renormalizable operators (NRO's). In principle, the determination of the complete set of such NRO's is a necessary first step in the analysis, since the renormalization group equations are affected when the fields with intermediate scale masses decouple. The determination of the complete set of such operators and the subsequent electroweak symmetry breaking patterns is beyond the scope of this paper (but is the subject of a future investigation).

\section{CONCLUSIONS}

This paper is the culmination of a program that sets out to derive the phenomenological implications of a class of quasirealistic string models. We have determined the observable sector gauge symmetry breaking patterns and the mass spectrum for a class of representative string-scale flat directions of a prototype model by merging the top-down approach (employing the string results for the effective superpotential couplings) and the bottom-up approach (adding the soft supersymmetry breaking mass parameters by hand).

The set of top-down inputs builds on the results of our previous work: (i) the classification of the $D$ flat directions of the model that cancel the anomalous $D$ term and can be proved to be $F$ flat to all orders [1] and (ii) the determination of the effective theory along such flat directions. In particular, we have utilized the results of [3], in which the complete mass spectrum and effective trilinear couplings of the observable sector were presented for two representative flat directions of model 5 of [9] (CHL5), which leave an additional $U(1)^{\prime}$ as well as the SM gauge group unbroken. The first representative flat direction has a minimal number of couplings of the observable sector fields, while the second flat direction has a richer structure of couplings, with implications for fermion textures.

The mass spectrum and couplings of these effective theories are not realistic, as expected. We found that in general there are a number of superfields which remain massless at the string scale, and that while the scalars acquire masses from the soft supersymmetry breaking terms, within our assumptions there is no mechanism for some of the fermions to acquire masses. The gauge coupling unification is not realistic (although better than expected due to the amount of additional matter superfields and the higher Kač-Moody level in the model), and the hidden sector gauge groups are not asymptotically free, thus disallowing an implementation of dynamical supersymmetry breaking scenarios. In addition, the effective trilinear couplings have a number of nonstandard features which were examined in detail in [3], such as the absence of a canonical effective $\mu$ term and the presence of baryon and lepton number violating couplings, as well as (potentially realistic) hierarchies of fermion masses.

Since the purpose of this program is to explore the general features of this class of quasi-realistic models systematically, and not to search for a specific, fully realistic model (an unlikely possibility), we continue the analysis by investigating the gauge symmetry breaking patterns and the low energy spectrum for the representative examples. However, this study requires the implementation of supersymmetry breaking, which we parametrize by soft supersymmetry breaking masses put in by hand at the string scale, due to the absence of a satisfactory scenario for supersymmetry breaking in 
string theory. This introduces free parameters in the effective theory, and thus the unique predictive power of a particular string vacuum is lost. In particular, the concrete results for the low energy spectrum depend on the initial conditions for the soft masses at the string scale.

We chose to analyze scenarios which lead to a realistic $Z-Z^{\prime}$ hierarchy; as argued on general grounds in $[13,14]$, the breaking scale of the $U(1)^{\prime}$ is at the electroweak scale or at an intermediate scale (if the symmetry breaking occurs along a $D$ flat direction). For each representative flat direction we determine the low energy spectrum explicitly for a typical choice of initial conditions that yield a realistic $Z-Z^{\prime}$ hierarchy. The emphasis was on the study of the Higgs sector, in order to contrast its features with that encountered in both the MSSM and string motivated models with an additional $U(1)^{\prime}$ [14]. In the symmetry breaking scenarios for these two representative examples, the novel feature is that the number of Higgs fields that participate in the symmetry breaking is larger than that assumed in [14] (there are at least three Higgs doublets and one SM singlet participating in the symmetry breaking process). In addition, the presence of some and absence of other non-canonical $\mu$ terms implies new patterns in the low energy mass spectrum.

For the first representative flat direction, the symmetry breaking scale of the $U(1)^{\prime}$ is at the electroweak scale, because all of the $U(1)^{\prime}$ charged singlets that remain massless at the string scale have charges of the same sign. To obtain a realistic $Z-Z^{\prime}$ hierarchy, we found it is necessary to have a nonminimal Higgs sector, with three Higgs doublets and one singlet (which has a large VEV). This scenario can be obtained with mildly nonuniversal soft supersymmetry breaking mass parameters at the string scale. We present the complete mass spectrum, including that of the supersymmetric partners, for a typical choice of initial conditions. The resulting mass spectrum at the electroweak scale includes massless and ultralight charginos, neutralinos, and Higgs bosons, due to the absence of enough canonical or non-canonical effective $\mu$ terms. In particular, the absence of an effective $\mu$ term involving one of the Higgs doublets provides an additional global $U(1)$ symmetry in the scalar potential that is spontaneously broken, resulting in a Goldstone boson present in the low energy spectrum. In addition, the mass of lightest neutral Higgs boson is controlled by the scale of the breakdown of this global symmetry, and thus obeys a different bound than the traditional bound in the MSSM.

For the second representative flat direction, the $U(1)^{\prime}$ breaking can be either at the electroweak scale or at an intermediate scale. Once again, the electroweak scale scenario requires an extended Higgs sector to obtain a $Z^{\prime}$ that is consistent with experiment. In addition, to study the fermion texture in the down-quark sector of this model, additional Higgs doublets are needed to acquire VEV's. In contrast to the previous model, there may be $C P$-violating phases in the Higgs sector of the model, though these are absent for the specific example considered. The electroweak scale symmetry breaking scenario is achievable with mildly nonuniversal boundary conditions. There is a fairly realistic $d: s: b$ mass hierarchy, although the absolute scale is much too high. The corresponding CKM matrix has no mixings of the first two families with the third, and an unrealistic maximal mixing between the first and second generations.

The intermediate scale symmetry breaking scenario can be achieved with mild nonuniversality of the soft supersymmetry breaking mass parameters, at a range of intermediate scales. It is purely radiative in origin, because of the absence of the relevant nonrenormalizable terms (and trilinear terms involving the heavy fields) in the superpotential. We plan to address the electroweak symmetry breaking in this scenario after decoupling the heavy fields, which requires a detailed analysis of a class of nonrenormalizable operators, in a future paper.

Although the analysis of the low energy implications for the representative examples studied in this paper reveals a number of additional unacceptable phenomenological consequences, it nevertheless demonstrates new features of the gauge symmetry breaking patterns, in particular that associated with the Higgs sector of the theory. The type of nonminimal extensions of the Higgs sector and their specific couplings, which we encountered in the analysis of the specific string models, should be of general interest in the phenomenological investigation of models beyond the MSSM, and thus deserves further study.

\section{ACKNOWLEDGMENTS}

We would like to thank J. Lykken for making available to us the program that generates the massless spectrum of free fermionic string vacua as well as the original program that calculates the superpotential couplings. L.E. acknowledges G. Kane for useful discussions. This work was supported in part by U.S. Department of Energy Grant No. DOE-EY-7602-3071.
[1] G. Cleaver, M. Cvetič, J.R. Espinosa, L. Everett, and P. Langacker, Nucl. Phys. B525, 3 (1998).

[2] G. Cleaver, M. Cvetič, J.R. Espinosa, L. Everett, and P. Langacker, hep-th/9805133.

[3] G. Cleaver, M. Cvetič, J.R. Espinosa, L. Everett, P. Langacker, and J. Wang, Phys. Rev. D 59, 055005 (1999).

[4] L. Ibáñez, J.E. Kim, H.P. Nilles, and F. Quevedo, Phys. Lett. B 191, 282 (1987); J.A. Casas and C. Muñoz, ibid. B209, 214
(1988); 214, 157 (1988); J.A. Casas, E. Katehou, and C. Muñoz, Nucl. Phys. B317, 171 (1989); A. Font, L. Ibáñez, H.P. Nilles, and F. Quevedo, Phys. Lett. B 210, 101 (1988); A. Chamseddine and M. Quirós, ibid. 212, 343 (1988); Nucl. Phys. B316, 101 (1989); A. Font, L. Ibáñez, F. Quevedo, and A. Sierra, ibid. B331, 421 (1990).

[5] B. Greene, K. Kirlin, P. Miron, and G.G. Ross, Nucl. Phys. B278, 667 (1986); B292, 606 (1987). 
[6] I. Antoniadis, J. Ellis, J. Hagelin, and D.V. Nanopoulos, Phys. Lett. B 231, 65 (1989); I. Antoniadis, G.K. Leontaris, and J. Rizos, ibid. 245, 161 (1990); A.E. Faraggi, Nucl. Phys. B387, 239 (1992); hep-th/9708112; A.E. Faraggi and D.V. Nanopoulos, Phys. Rev. D 48, 3288 (1993).

[7] A. Faraggi, D.V. Nanopoulos, and K. Yuan, Nucl. Phys. B335, 347 (1990); A. Faraggi, Phys. Rev. D 46, 3204 (1992).

[8] A. Faraggi, Phys. Lett. B 278, 131 (1992); Nucl. Phys. B403, 101 (1993); Phys. Lett. B 339, 223 (1994).

[9] S. Chaudhuri, G. Hockney, and J. Lykken, Nucl. Phys. B469, 357 (1996).

[10] K.R. Dienes, Phys. Rep. 287, 447 (1997).

[11] M. Cvetič, L. Everett, and J. Wang, Phys. Rev. D (to be published), hep-ph/9808321.

[12] M. Cvetič, L. Everett, and J. Wang, Nucl. Phys. B538, 52 (1999).

[13] M. Cvetič and P. Langacker, Phys. Rev. D 54, 3570 (1996); Mod. Phys. Lett. A 11, 1247 (1996); in Perspectives on Supersymmetry, edited by G. Kane (World Scientific, Singapore, 1998), hep-ph/9707451.
[14] M. Cvetič, D.A. Demir, J.R. Espinosa, L. Everett, and P. Langacker, Phys. Rev. D 56, 2861 (1997). For related work, see Refs. [15-18].

[15] G. Cleaver, M. Cvetič, J.R. Espinosa, L. Everett, and P. Langacker, Phys. Rev. D 57, 2701 (1998).

[16] D. Suematsu and Y. Yamagishi, Int. J. Mod. Phys. A 10, 4521 (1995).

[17] P. Langacker and J. Wang, Phys. Rev. D 58, 115010 (1998).

[18] E. Keith and E. Ma, Phys. Rev. D 56, 7155 (1997).

[19] V. Kaplunovsky, Nucl. Phys. B307, 145 (1988); B382, 436(E) (1992).

[20] L. Dixon and M. Cvetič (unpublished); M. Cvetič, Phys. Rev. Lett. 59, 2829 (1987); D. Bailin, D. Dunbar, and A. Love, Phys. Lett. B 219, 76 (1989); S. Kalara, J. López, and D.V. Nanopoulos, ibid. B245, 421 (1990); Nucl. Phys. B353, 650 (1991); A. Faraggi, ibid. B487, 55 (1997).

[21] P. Langacker and N. Polonsky, Phys. Rev. D 49, 1454 (1994); M. Carena et al., Nucl. Phys. B426, 269 (1994); V. Barger, M.S. Berger, and P. Ohmann, Phys. Rev. D 47, 1093 (1993).

[22] D. Comelli and J.R. Espinosa, Phys. Lett. B 388, 793 (1996); J.R. Espinosa, Surv. High Energy Phys. 10, 279 (1997). 